\title{
Manifestly covariant classical correlation dynamics II. Transport equations and Hakim equilibrium conjecture
}

\author{
Chushun Tian \\ Institut für Theoretische Physik, Zülpicher Str. 77, Köln, D-50937, Germany
}

(Dated: November 22, 2018)

\begin{abstract}
This is the second of a series of papers on the special relativistic classical statistical mechanics. Employing the general theory developed in the first paper we rigorously derive the relativistic Vlasov, Landau and Boltzmann equation. The latter two advocate the Jütner distribution as the equilibrium distribution. We thus, at the full microscopic level, provide a support for the recent numerical finding [D. Cubero et. al., Phys. Rev. Lett. 99, 170601 (2007)] of the special relativistic generalization of the Maxwell-Boltzmann distribution. Furthermore, the present theory allows us to rigorously calculate various correlation functions at the relativistic many-body equilibrium. Therefore, the relativistic many-body equilibrium conjecture of Hakim is justified.
\end{abstract}

PACS numbers: 03.30.+p, 52.25.Dg

\section{INTRODUCTION}

The kinetic theory is one of the pillars of studies of relativistic transport phenomena in various systems ranging from star clusters or galaxies 1, 2, 3, , 4] to plasmas in fusion [5], quantum chromodynamics [6, 7] and graphene discovered very recently [8]. The manifestly covariant counterparts of various classical transport equations, such as the kinetic equation of Vlasov, Fokker-Planck, Landau and Boltzmann, were proposed long time ago (for a review see, for example, Ref. 9]), and their applications nowadays have been well documented 10, 11]. These manifestly covariant transport equations have received justifications from various microscopic approaches [2, 3, 9, 12, 13, 14, 15]. It is rather typical in rederiving relativistic transport equations (for examples, see 9, 13, 15]) that the Liouvillian dynamics of complete many-body distribution function is bypassed and, crucially, the truncation approximation is resorted to. Therefore, despite of the great success of this kind of microscopic approaches [9, 13, 15] a fundamentally important problem remains unsolved. That is, will a manifestly covariant transport equation be compatible with the Liouvillian dynamics of the complete many-body distribution function? This issue has been addressed continuously for several decades by many workers [2, 3, $9,12,16,17,18,19]$ and been far less advanced. This is by no means of pure theoretical interests, rather, may find considerable practical applications. Indeed, experiences in the Newtonian physics have shown that to go beyond the weak coupling and Markovian approximation is inevitable in order to understand collective dielectric effects in electromagnetic plasmas [20] and the infrared divergence of the Fokker-Planck equation describing the stellar dynamics 21, 22]. In an insightful paper Kandrup first realized that a Liouville equation of the complete many-body distribution function admits an exact closed (nonlinear) kinetic equation which is satisfied by the reduced one-body distribution function and manifestly covariant [3]. Various special transport equations, remarkably, may be unified within this general kinetic equation.
Unfortunately, he encountered principal difficulties when proceeded further to derive the general kinetic equation explicitly. Such a big gap is filled in the first of this series of papers (denoted as Paper I) by the developed manifestly covariant classical correlation dynamics [23]. One of the main subjects of the present paper is to recover various special transport equations systematically from the exact, manifestly covariant and closed kinetic equation 24] found in Paper I, which we call the general transport equation.

The existence of the general transport equation has important implications on several aspects of classical special relativistic (nonequilibrium) statistical mechanics which are undergoing intensified studies. First of all, the mathematical structure possessed by the general transport equation is in excellent agreement with various approximate transport equations obtained by other microscopic approaches [2, 3, 12, 13, 14, 15, 18, 25], but remarkably different from that proposed by Horwitz and coworkers 17]. Recently triggered by the latter there have been many scientific activities [26, 27] searching for the special relativistic generalization of the Maxwell-Boltzmann distribution. The general transport equation reinforces the concept well established by approximate transport equations. That is, the Jüttner distribution serves as the relativistic (one-body) equilibrium in dilute systems. Therefore, it is suggested that other alternatives to the Jüttner equilibrium 17] might be specific to the (deterministic) relativistic many-body dynamics.

Secondly, in past years the relativistic Brownian motion and diffusion have experienced considerable conceptual developments and found important practical applications [28]. Long time ago it was known that in the Minkowski spacetime nontrivial Lorentz invariant Markovian processes do not exist [29, 30]. It turns out that the relativistic Brownian motion is interpreted as relativistic Markovian processes in the $\mu$ phase space, and the latter is completely described by a relativistic Fokker-Plancktype equation. So far these observations have been investigated thoroughly at the level of one-particle physics [28], and Paper I is the first to substantiate these impor- 
tant observations at the level of the genuine relativistic many-body physics. Indeed, the general transport equation arises from (i) the thermodynamic limit namely the particle number $N \rightarrow+\infty$ and, (ii) that at given (global) proper time particles lose the memory of the history of the entire system. The condition (ii) is in sharp contrast to the deterministic relativistic many-body dynamics, where the particle interaction is profoundly nonlocal in spacetime, and serves as the many-body dynamical origin of the Markovian processes in the $\mu$ phase space. In particular, in Paper I the proper time parametrized equation in the 8-dimensional $\mu$ phase space is rigorously justified, which was first obtained by Hakim [30], and important roles of which have very recently been reinforced 31]. From these perspectives the theory presented in this series of papers may be considered as a microscopic approach to (classical) special relativistic Brownian motion complementary to the one based on the relativistic Langevin equation. In particular, it might be proven to be a useful technique in exploring the concepts such as relativistic noises, friction and fluctuation theorem.

There is an adjacent important yet unsolved problem that may be explored in the present theoretical scope. That is, to formulate the relativistic many-body equilibrium. In the notable critical analysis [9] Hakim conjectured that at equilibrium there might exist an infinite Lorentz invariant hierarchy of correlation functions which is invariant under the spacetime translation and is merely determined by the Jüttner distribution. Unfortunately, further progresses have been impeded by the truncation approximation intrinsic to various microscopic approaches (for examples, Refs. [9, 15]), and the (dis)proof so far has been missing. The general principles given in Paper I paves the way towards justifying this conjecture. There the hierarchy of (physical) correlation functions is found explicitly, which is merely determined by the (physical) one-body distribution function. Provided that the general transport equation admits an equilibrium distribution, then the hierarchy of equilibrium correlation functions is uniquely determined. To carry out this program for rarified electromagnetic plasmas constitutes another main subject of the present paper.

The present paper is written in the self-contained manner. The readers that would not like to access to the mathematical foundation may skip Paper I. The paper is organized as follows: Sec. [II is an exposition of the main results of Paper I, and the exact starting point of the present paper is pointed out. The rest is devoted to applications in relativistic plasmas with electromagnetic interactions. In Sec. III we derive the relativistic Vlasov, Landau and Boltzmann equation from the general transport equation found in Paper I. In Sec. IV we show that the present theory fully agrees with the recent numerical finding [26] and advocates the Jüttner distribution as the special relativistic generalization of the MaxwellBoltzmann distribution. The Hakim equilibrium conjecture is justified, and the two-body equilibrium correlation function is exactly calculated. We conclude this series of papers in Sec. V] Some technical details are given in Appendix $\mathrm{A}, \mathrm{C}$

Finally we list some of the notations and conventions. We choose the unit system with the speed of light $c=1$. To distinguish from the Minkowski 4-vector we use the bold font to denote the vector in the Euclidean space. Greek indices running from 0 to 3 are further used to denote the component of the former. The Einstein summation convention is applied to these indices. The 4dimensional Minkowski space is endowed with the metric $\eta^{\mu \nu}=\operatorname{diag}(1,-1,-1,-1)$. The scalar product of two 4 -vectors is defined as $a \cdot b \equiv \eta^{\mu \nu} a_{\mu} b_{\nu}=a_{\mu} b^{\mu}$. In particular, $a \cdot a \equiv a^{2}$. In addition to the usual mathematical symbols we use the following notations:

$$
\begin{array}{ll}
\partial_{\mu}, & \text { covariant derivative: } \partial_{\mu}=\partial / \partial x^{\mu} ; \\
d^{4} z, & \text { volume element in } 4 \text {-dimensional Minkowski } \\
& \text { space: } d^{4} z=d z^{0} d z^{1} d z^{2} d z^{3} ; \\
d^{3} \mathbf{z}, & \text { volume element in } 3 \text {-dimensional Euclidean } \\
& \text { space: } d^{3} \mathbf{z}=d z^{1} d z^{2} d z^{3} ; \\
\delta^{(d)}(f), & d \text {-dimensional Dirac function: } \\
\theta(x), & \text { Heaviside function; } \\
d \Sigma_{\mu}, & \text { differential form of spacelike } 3 \text {-surface: } \\
& d \Sigma_{\mu}=\frac{1}{3 !} \epsilon_{\mu \nu \rho \lambda} d x^{\nu} \wedge d x^{\rho} \wedge d x^{\lambda} \text { with } \epsilon_{\mu \nu \rho \lambda} \\
& \text { being } \pm 1 \text { when }(\mu \nu \rho) \text { is an even }(\text { odd }) \\
& \text { permutation of }(0123) \text { and being } 0 \text { otherwise } \\
x_{i}[\varsigma], & \text { world line of particle } i ; \\
x_{i}(\varsigma), & \text { 4-position of particle } i \text { at proper time } \varsigma .
\end{array}
$$

\section{MAIN RESULTS OF GENERAL THEORY}

This section is devoted to presenting the exact starting point that underlies the entire analysis of the following sections. For this purpose we first briefly review the manifestly covariant correlation dynamics developed in Paper I, and introduce all the mathematical objects used throughout this paper. It should be stressed that at each step of the manipulations below the manifest covariance is preserved.

\section{A. Correlation dynamics of $\tau$-parametrized evolution}

Consider a microscopic system composed of $N$ identical (classical) particles with mass $m$, the dynamics of which is formulated within the action-at-a-distance formalism [32]. More precisely, the history of the system is described by a bundle of $N$ particle world lines which solve the following relativistic motion equations:

$$
\begin{aligned}
\frac{d x_{i}^{\mu}}{d \tau_{i}} & =\frac{p_{i}^{\mu}}{m} \equiv u_{i}^{\mu} \\
\frac{d p_{i}^{\mu}}{d \tau_{i}} & =\sum_{j \neq i}^{N} F_{i j}^{\mu}\left(x_{i}, p_{i}\right) .
\end{aligned}
$$

Here $x_{i}^{\mu}\left(\tau_{i}\right), u_{i}^{\mu}\left(\tau_{i}\right), p_{i}^{\mu}\left(\tau_{i}\right)$ are the 4-position, the 4velocity and the 4 -momentum vector of particle $i$ de- 
pending on the proper time $\tau_{i}$, respectively, and $F_{i j}^{\mu}$ is the force acting on particle $i$ by particle $j$. Notice that $F_{i j}^{\mu}\left(x_{i}, p_{i}\right)$ functionally depends on the world line of particle $j$ namely $x_{j}\left[\tau_{j}\right]$. For simplicity we here consider the case where the external force is absent, and the interacting force $F_{i j}^{\mu}$ is conservative, i.e.,

$$
\frac{\partial}{\partial p_{i}^{\mu}} F_{i j}^{\mu}\left(x_{i}, p_{i}\right)=0 .
$$

The mass-shell constraint: $p_{i}^{2}=m^{2}$ is preserved by

$$
p_{i} \cdot F_{i j}\left(x_{i}, p_{i}\right)=0 .
$$

Eqs. (11) and (2) suggest that in order to formulate a statistical theory of an ensemble of such systems the introduction of an $8 N$-dimensional $\Gamma$ phase space and the associated probability (phase) density function $\mathcal{D}$ is required. (The mass-shell constraint is absorbed into the distribution function.) Remarkably, this distribution function differs from its Newtonian counterpart in that it is parametrized by $N$ (rather than 1) proper times, and functionally depends on $N$ particle world lines. For $\mathcal{D}$ the probability conservation law gives $N$ manifestly covariant Liouville equations. By further introducing an auxiliary "gauge" condition-to demand the $N$ proper times to change uniformly-we obtain a manifestly covariant single-time Liouville equation of $\mathcal{D}\left(x_{1}, p_{1}, \tau_{1}+\tau, \cdots, x_{N}, p_{N}, \tau_{N}+\tau ; x_{1}[\varsigma], \cdots, x_{N}[\varsigma]\right):$

$$
\left(\frac{\partial}{\partial \tau}-\hat{\mathfrak{L}}\right) \mathcal{D}=0,
$$

where the Liouvillian $\hat{\mathfrak{L}}$ is given by

$$
\begin{aligned}
\hat{\mathfrak{L}} & =\hat{\mathfrak{L}}^{0}+\lambda \hat{\mathfrak{L}}^{\prime}, \\
\hat{\mathfrak{L}}^{0} & =-\sum_{i=1}^{N} u_{i}^{\mu} \partial_{\mu i}, \quad \lambda \hat{\mathfrak{L}}^{\prime}=\sum_{i<j} \lambda \hat{\mathcal{L}}_{i j}^{\prime}, \\
\lambda \hat{\mathcal{L}}_{i j}^{\prime} & \equiv-\left\{F_{i j}^{\mu}\left(x_{i}, p_{i}\right) \frac{\partial}{\partial p_{i}^{\mu}}+F_{j i}^{\mu}\left(x_{j}, p_{j}\right) \frac{\partial}{\partial p_{j}^{\mu}}\right\},
\end{aligned}
$$

with $\hat{\mathfrak{L}}^{0}$ and $\lambda \hat{\mathcal{L}}_{i j}^{\prime}$ the free and the two-body interacting Liouvillian, respectively. Notice that here the dimensionless parameter $\lambda$ characterizes the interaction strength.

For the evolution parametrized by $\tau$ namely Eq. (5) the correlation dynamics analysis may be performed for a large class of realistic systems 23]. First, we define the following distribution vector:

$$
\overrightarrow{\mathfrak{D}} \equiv\left(\left\{\mathcal{D}_{1}\right\},\left\{\mathcal{D}_{2}\right\}, \cdots,\left\{\mathcal{D}_{N}\right\} \equiv \mathcal{D}\right),
$$

where the reduced $s$-body distribution function $\mathcal{D}_{s}$ is obtained by integrating out arbitrary $(N-s)$ particle phase coordinates and because of this for each $\left\{\mathcal{D}_{s}\right\}$ there are $N ! /[(N-s !) s !]$ components. With the help of this definition the BBGKY hierarchy is rewritten in a compact form:

$$
\left(\frac{\partial}{\partial \tau}-\hat{\mathfrak{L}}\right) \overrightarrow{\mathfrak{D}}=0
$$

Eqs. (7) and (8) constitute the reduced distribution function representation of the single-time Liouville equation (5). To proceed further we introduce the so-called correlation pattern representation allowing a more delicate decomposition of the distribution vector or more general functions. A correlation pattern, denoted as $\left|\Gamma_{s}\right\rangle$ (or $\left\langle\Gamma_{s}\right|$ ), describes the statistical correlation of given $s$-particle group. More precisely, consider an $s$-particle group $\left(i_{1} \cdots i_{s}\right), s \leq N$, then the correlation pattern is generally given by $\left.\left|\mathrm{P}_{1}\right| \mathrm{P}_{2}|\cdots| \mathrm{P}_{\mathrm{j}}\right\rangle$ (or $\left\langle\mathrm{P}_{1}\left|\mathrm{P}_{2}\right| \cdots\left|\mathrm{P}_{\mathrm{j}}\right|\right.$ ), where $\mathrm{P}_{1}, \cdots, \mathrm{P}_{j}$ is a partition of $\left(i_{1} \cdots i_{s}\right)$. It implies that in the statistical sense within $\mathrm{P}_{i}, 1 \leq i \leq j$ the particles correlate with each other, while the particle groups $\mathrm{P}_{i}, 1 \leq i \leq j$ are independent. The reduced $s$-body distribution function in this presentation now reads out as $\mathcal{D}_{s}=\sum_{\Gamma_{s}}\left|\Gamma_{s}\right\rangle\left\langle\Gamma_{s}\right| \mathcal{D}_{s}$ which is none but the cluster expansion. It should be stressed, however, that it differs from a traditional one [33, 34] in that the distribution functions depend on the particle world lines. This is, indeed, an important ingredient of the Klimontovich technique in the Newtonian context [13] and was generalized to the special relativity-in a manifestly covariant manner-by Hakim [9].

A particularly important correlation pattern is the socalled vacuum state: $\left|\Gamma_{s}\right\rangle \equiv\left|0_{s}\right\rangle$ (or $\left.\left\langle\Gamma_{s}\right| \equiv\left\langle 0_{s}\right|\right)$, where the given $s$ particles are (statistically) independent. In contrast, all the other correlation patterns are called correlation states. With this definition the vacuum and the correlation operator, denoted as $\mathbf{V}$ and $\mathbf{C}$, respectively, are defined as follows:

$$
\mathbf{V}\left|\Gamma_{r}\right\rangle=\delta_{0_{r} \Gamma_{r}}\left|\Gamma_{r}\right\rangle, \quad \mathbf{C}\left|\Gamma_{r}\right\rangle=\left(1-\delta_{0_{r} \Gamma_{r}}\right)\left|\Gamma_{r}\right\rangle,
$$

which project given functions onto the vacuum or the correlation state.

Then, a series of rigorous theorems establish the following important properties. First of all, the distribution vector $\overrightarrow{\mathfrak{D}}$ is split into the kinetic component $\hat{\Pi}_{\mathrm{k} .} \overrightarrow{\mathfrak{D}}$ and the nonkinetic component $\hat{\Pi}_{\text {n.k. }} \overrightarrow{\mathfrak{D}}$, i.e.,

$$
\overrightarrow{\mathfrak{D}}=\hat{\Pi}_{\mathrm{k}} \cdot \overrightarrow{\mathfrak{D}}+\hat{\Pi}_{\mathrm{n} \cdot \mathrm{k} \cdot} \overrightarrow{\mathfrak{D}}
$$

and the latter is irrelevant for large global proper times. The former is further decomposed into the vacuum and the correlation state, i.e.,

$$
\hat{\Pi}_{\mathrm{k} .} \overrightarrow{\mathfrak{D}}=\mathbf{V} \hat{\Pi}_{\mathrm{k} .} \overrightarrow{\mathfrak{D}}+\mathbf{C} \hat{\Pi}_{\mathrm{k}} \cdot \overrightarrow{\mathfrak{D}}
$$

and the correlation state is fully determined by the vacuum state. In the thermodynamic limit $N \rightarrow+\infty$ as written in the correlation pattern representation the evolution of $\mathbf{V} \hat{\Pi}_{\mathrm{k}} \overrightarrow{\mathfrak{D}}$ and $\mathbf{C} \hat{\Pi}_{\mathrm{k}} \cdot \overrightarrow{\mathfrak{D}}$ are expressed in terms of two infinite equation hierarchies. It is remarkable that both infinite equation hierarchies are determined merely by a reduced one-body distribution function 
$\tilde{\mathcal{D}}\left(x, p ; \mathcal{X}_{(x, p)}\right)$ which solves the exact closed equation:

$$
\begin{aligned}
& \left\{\frac{\partial}{\partial \tau}+u_{1}^{\mu} \partial_{\mu 1}-\int d 2 \lambda \hat{\mathcal{L}}_{12}^{\prime} \tilde{\mathcal{D}}\left(2 ; \mathcal{X}_{2}\right)\right\} \tilde{\mathcal{D}}\left(1 ; \mathcal{X}_{1}\right) \\
= & \sum_{j \geq 2} \int d 2 \cdots \int d j\langle 1|\mathbf{V}(\boldsymbol{\Gamma}-\hat{\mathfrak{L}}) \mathbf{V}| 1|\cdots| j\rangle \prod_{s=1}^{j} \tilde{\mathcal{D}}\left(s ; \mathcal{X}_{s}\right) .
\end{aligned}
$$

Here we have introduced the shorthand notations: $\tilde{\mathcal{D}}\left(i ; \mathcal{X}_{i}\right) \equiv \tilde{\mathcal{D}}\left(x_{i}, p_{i} ; \mathcal{X}_{\left(x_{i}, p_{i}\right)}\right)$ and $d i \equiv d^{4} x_{i} d^{4} p_{i}$, and the notation: $\mathcal{X}_{(x, p)}$ stands for some world line passing through the phase point $(x, p)$. Notice that the twobody interacting Liouvillian $\lambda \hat{\mathcal{L}}_{i j}^{\prime}\left(x_{i}, p_{i} ; x_{j}, p_{j}\right)$ is a functional of the world lines $\mathcal{X}_{\left(x_{i}, p_{i}\right)}$ and $\mathcal{X}_{\left(x_{j}, p_{j}\right)}$. The operator $\mathbf{V} \boldsymbol{\Gamma V}$ determines the evolution of the vacuum state $\mathbf{V} \hat{\Pi}_{k} \cdot \overrightarrow{\mathfrak{D}}$, and is given by the following functional equation:

$$
\begin{aligned}
& \mathbf{V} \boldsymbol{\Gamma} \mathbf{V}=\mathbf{V} \hat{\mathfrak{L}} \mathbf{V}+\int_{0}^{\infty} d s \mathbf{V} \hat{\mathfrak{g}}(s) \mathbf{V} \exp (-s \mathbf{V} \boldsymbol{\Gamma} \mathbf{V}) \\
& \mathbf{V} \hat{\mathfrak{g}}(s) \mathbf{V}=\int_{C} \frac{d z}{2 \pi} e^{-i z s} \mathbf{V} \hat{\mathcal{E}}(z) \hat{\mathfrak{R}}^{0}(z) \mathbf{C} \lambda \hat{\mathfrak{L}}^{\prime} \mathbf{V}
\end{aligned}
$$

where the contour $C$ (in the complex plane) lies above all the singularities of the Laplace transform of $\mathcal{D}$, and

$$
\begin{aligned}
\hat{\mathcal{E}}(z) & =\sum_{n=0}^{\infty} \lambda^{n+1} \hat{\mathfrak{L}}^{\prime}\left\{\mathbf{C} \hat{\mathfrak{R}}^{0}(z) \hat{\mathfrak{L}}^{\prime}\right\}^{n}, \\
\hat{\mathfrak{R}}^{0}(z) & =\frac{1}{-i z-\hat{\mathfrak{L}}^{0}} .
\end{aligned}
$$

To our best knowledge a kinetic equation similar to Eq. (12) was first obtained by Hakim by using the weak coupling approximation [9]. A simplified equation of Hakim has very recently been found to play important roles in studies of the relativistic Brownian motion [31].

\section{B. General transport equation and hierarchy of physical correlation functions}

The $\tau$-parametrized evolution may not be observable because $\mathcal{D}$ is normalized in the $8 N$-dimensional phase space. Rather, to match macroscopic observations the following physical distribution function:

$$
\mathcal{N}\left(x_{1}, p_{1}, \cdots, x_{N}, p_{N} ; x_{1}[\varsigma], \cdots, x_{N}[\varsigma]\right) \equiv \int \prod_{i=1}^{N} d \tau_{i} \mathcal{D}(16)
$$

is introduced which may be considered formally as the stationary solution to Eq. (5). As a result, the nonkinetic component of $\mathcal{N}$ identically vanishes. Moreover, in the thermodynamic limit $N \rightarrow$ $+\infty$ both hierarchies (corresponding to the vacuum and the correlation state, respectively) is determined merely by a physical one-body distribution function $f(x, p)$. Here by "physical" the normalization condition: $\lim _{N \rightarrow+\infty} N^{-1} \int_{\Sigma \otimes U^{4}} d \Sigma_{\mu} d^{4} p u^{\mu} f(x, p)=1$ is implied, where $\Sigma$ is a spacelike 3 -surface, and $U^{4}$ is the 4-dimensional Minkowski momentum space.

The stationary solution to Eq. (12) results in a general transport equation which is manifestly covariant and closed:

$$
\left\{u_{1}^{\mu} \partial_{\mu 1}-\int_{\Sigma_{2} \otimes U_{2}^{4}} d \Sigma_{\mu 2} d^{4} p_{2} u_{2}^{\mu} \lambda \hat{\mathcal{L}}_{12}^{\prime} f(2)\right\} f(1)=\mathbb{K}[f],
$$

where $f(i)$ is the shorthand notation of $f\left(x_{i}, p_{i}\right)$, and the collision integral is given by

$$
\begin{aligned}
\mathbb{K}[f]=\sum_{j \geq 2} & \int_{\Sigma_{2} \otimes U_{2}^{4}} d \Sigma_{\mu 2} d^{4} p_{2} u_{2}^{\mu} \cdots \int_{\Sigma_{j} \otimes U_{j}^{4}} d \Sigma_{\mu j} d^{4} p_{j} u_{j}^{\mu} \\
& \times\langle 1|\mathbf{V}(\mathbf{\Gamma}-\hat{\mathfrak{L}}) \mathbf{V}| 1|\cdots| j\rangle \prod_{i=1}^{j} f(i)
\end{aligned}
$$

The solution to Eqs. (17) and (18), in turn, uniquely determines the hierarchy of physical correlation functions. More precisely, given an arbitrary $j$-particle correlation pattern $\Gamma_{j}(1, \cdots, j) \neq 0_{j}$, in the thermodynamic limit $N \rightarrow+\infty$ the physical correlation function (denoted as $\left.\left\langle\Gamma_{j}\right| \overrightarrow{\mathfrak{N}}_{\infty}\right)$ reads out as

$$
\begin{aligned}
& \left\langle\Gamma_{j}\right| \overrightarrow{\mathfrak{N}}_{\infty} \\
= & \int_{0}^{\infty} d s \int_{0}^{s} d s^{\prime} \sum_{n=j}^{\infty} \prod_{i=j+1}^{n} \int_{\Sigma_{i} \otimes U_{i}^{4}} d \Sigma_{\mu i} d^{4} p_{i} u_{i}^{\mu} \prod_{k=1}^{n} f(k) \\
& \times\left\langle\Gamma_{j}\left|\mathbf{C} \hat{\mathfrak{U}}^{0}\left(s-s^{\prime}\right) \hat{\mathfrak{E}}\left(s^{\prime}\right) \mathbf{V} \exp (-s \mathbf{V} \mathbf{\Gamma V})\right| 1|\cdots| n\right\rangle,
\end{aligned}
$$

(The integration procedure: $\prod_{i=j+1}^{n} \int_{\Sigma_{i} \otimes U_{i}^{4}} d \Sigma_{\mu i} d^{4} p_{i} u_{i}^{\mu}$ is defined as unity for $n=j$.) where

$\hat{\mathfrak{U}}^{0}(\tau)=\int_{C} \frac{d z}{2 \pi} e^{-i z \tau} \hat{\mathfrak{R}}^{0}(z), \quad \hat{\mathfrak{E}}(\tau)=\int_{C} \frac{d z}{2 \pi} e^{-i z \tau} \hat{\mathcal{E}}(z)$.

In practical applications a perturbative expansion with respect to $\lambda$ may be further performed for the collision integral $\mathbb{K}[f]$ and the physical correlation function $\left\langle\Gamma_{j}\right| \overrightarrow{\mathfrak{N}}_{\infty}$. Such an expansion is represented by the diagrams constructed out of the free propagator and the interaction vertex (see the inset of Fig. 1). For the former the matrix element reads out as

$$
\left\langle i^{\prime}\left|e^{-\tau u_{j}^{\mu} \partial_{\mu j}}\right| i\right\rangle=\delta_{i j} \delta_{i i^{\prime}} e^{-\tau u_{i}^{\mu} \partial_{\mu i}} .
$$

For the latter there are two types: For the first type (the middle in the inset of Fig. 1) a particle joining the vertex from the right is annihilated (the dashed line), and the matrix element reads out as

$$
\begin{aligned}
& \left\langle i\left|\lambda \hat{\mathcal{L}}_{i^{\prime} j^{\prime}}^{\prime}\right| \Gamma_{2}(i, j)\right\rangle \\
= & \left(\delta_{i i^{\prime}} \delta_{j j^{\prime}}+\delta_{i j^{\prime}} \delta_{j i^{\prime}}\right) \int_{\Sigma_{j} \otimes U_{j}^{4}} d \Sigma_{\mu j} d^{4} p_{j} u_{j}^{\mu} \lambda \hat{\mathcal{L}}_{i j}^{\prime}
\end{aligned}
$$


(a)


(b)
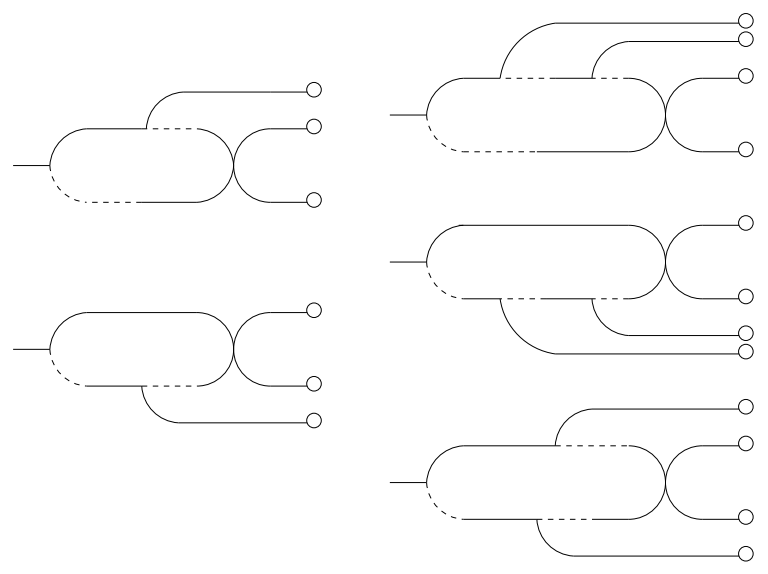

FIG. 1: Diagrams representing the perturbative expansion of the collision integral: (a) the weak coupling approximation and (b) the lowest order ring approximation. The circle stands for the physical one-body distribution function. Inset: free propagator (top) and two types of interaction vertex (middle and bottom).

with $\left|\Gamma_{2}(i, j)\right\rangle=|i j\rangle$ or $\left.|i| j\right\rangle$. For the second type (the bottom in the inset of Fig. 11) no particles (joining the vertex from the right) are annihilated. "Switching on" the interaction (at the vertex) introduces the (statistical) correlation, i.e., $\left\langle\Gamma_{2}(i, j)\right|=\langle i j|$ irrespective of the "initial" correlation pattern (to the right of the vertex), i.e., $\left|\Gamma_{2}(i, j)\right\rangle$. The matrix element for this type of the interaction vertex reads out as

$$
\left\langle i j\left|\lambda \hat{\mathcal{L}}_{i^{\prime} j^{\prime}}^{\prime}\right| \Gamma_{2}(i, j)\right\rangle=\left(\delta_{i i^{\prime}} \delta_{j j^{\prime}}+\delta_{i j^{\prime}} \delta_{j i^{\prime}}\right) \lambda \hat{\mathcal{L}}_{i j}^{\prime} .
$$

Eqs. (17)-(19) justify the manifestly covariant Bogoliubov functional assumption [33, 34, 35]. They constitute the complete set for describing various physical phenomena such as transport processes, (macroscopic) relativistic hydrodynamics, and (physical) correlations at equilibrium, and serve as the exact starting point of subsequent sections. The remaining of this paper is, indeed, devoted to the applications of Eqs. (17)-(19) in classical relativistic plasmas with electromagnetic interactions.

\section{SPECIAL TRANSPORT EQUATIONS}

In this section we will consider a rarified electron plasma that is near the local equilibrium with the density and the temperature in the local rest frame as $\rho_{0}$ and $T$, respectively. We will justify that various (namely
Vlasov [9], Landau [13, 36] and Boltzmann [10, 11]) manifestly covariant kinetic equations existing in literatures are unified within the general transport equation (17). Particular attention will be paid to the additional approximations made. In doing so we expect to clarify the context where they are applicable.

It should be stressed that given a density $\rho_{0}$ the present theory is applicable for moderate temperatures. For sufficiently high or low temperatures quantum statistics and QED processes dominate, and the complete treatment requires a quantum theory. Let us now estimate such a condition. On one hand, two classical electrons may approach each other up to a distance $\sim e^{2} /\left(k_{B} T\right)$, where $k_{B}$ is the Boltzmann constant. In order for a classical scattering theory to be applicable it must be larger than the thermal deBroglie wavelength $\hbar / \sqrt{m k_{B} T}$. Consequently, we find $k_{B} T \ll \alpha^{2} m$ with $\alpha=\frac{1}{137}$ the fine structure constant. On the other hand, the plasma become degenerate when the thermal deBroglie wavelength and the mean distance $\sim \rho_{0}^{-1 / 3}$ are comparable. This implies that the quantum statistics can be ignored only for sufficiently high temperatures such that $\hbar / \sqrt{m k_{B} T} \ll \rho_{0}^{-1 / 3}$, i.e., $k_{B} T \gg \hbar^{2} \rho_{0}^{2 / 3} / m$. Thus, the temperature region for a classical theory to be applicable is

$$
\frac{\hbar^{2} \rho_{0}^{2 / 3}}{m} \ll k_{B} T \ll \alpha^{2} m .
$$

Notice that the inequality above imposes a restriction on the density, i.e., $\rho_{0} \ll\left(e^{2} m / \hbar^{2}\right)^{3}$. This implies that the fermi energy namely $\hbar^{2} \rho_{0}^{2 / 3} / m$ is the lowest energy scale and, in particular, is much smaller than the (classical) electromagnetic energy $e^{2} \rho_{0}^{1 / 3}$. The inequality (24) is the rigorous condition for the present classical theory to be applicable.

\section{A. General scheme}

Let us first prove an exact relation between the collision integral $\mathbb{K}[f]$ and the physical two-body correlation function. For the latter setting $\left\langle\Gamma_{j}\right|$ to be $\langle 12|$ for the hierarchy (19) we find

$$
\begin{aligned}
& \langle 12| \overrightarrow{\mathfrak{N}}_{\infty} \\
= & \int_{0}^{\infty} d s \int_{0}^{s} d s^{\prime} \sum_{n=j}^{\infty} \prod_{i=j+1}^{n} \int_{\Sigma_{i} \otimes U_{i}^{4}} d \Sigma_{\mu i} d^{4} p_{i} u_{i}^{\mu} \prod_{k=1}^{n} f(k) \\
& \times\left\langle 12\left|\mathbf{C} \hat{\mathfrak{U}}^{0}\left(s-s^{\prime}\right) \hat{\mathfrak{E}}\left(s^{\prime}\right) \mathbf{V} \exp (-s \mathbf{V} \mathbf{\Gamma V})\right| 1|\cdots| n\right\rangle .
\end{aligned}
$$

Notice that

$$
\mathbf{V}(\boldsymbol{\Gamma}-\hat{\mathfrak{L}}) \mathbf{V}=
$$

$\int_{0}^{\infty} d s \int_{0}^{s} d s^{\prime} \mathbf{V} \lambda \hat{\mathfrak{L}}^{\prime} \mathbf{C} \hat{\mathfrak{U}}^{0}\left(s-s^{\prime}\right) \hat{\mathcal{E}}\left(s^{\prime}\right) \mathbf{V} \exp (-s \mathbf{V} \boldsymbol{\Gamma} \mathbf{V})$.

Inserting it into Eq. (18) gives

$$
\mathbb{K}[f]=\int_{\Sigma_{2} \otimes U_{2}^{4}} d \Sigma_{\mu 2} d^{4} p_{2} u_{2}^{\mu}\left\langle 1\left|\lambda \hat{\mathfrak{L}}^{\prime}\right| 12\right\rangle\langle 12| \overrightarrow{\mathfrak{N}}_{\infty}
$$


This exact relation indicates that the partition of full distribution functions in the correlation pattern representation preserves the cluster expansion, which is guaranteed by the Clavin theorem in the Newtonian physics [37].

We remark that from Eq. (27) the collision integral is locally well defined provided that either interactions or (statistical) correlations are short-ranged in the spacelike 3-surface passing through $x_{1}$ and $x_{2}$. A relativistic plasma with electromagnetic interactions, indeed, belongs to the latter case. There, although the static transverse electromagnetic field is long-ranged, the Debye screening of the longitudinal electromagnetic field, as we will show in Sec. [V] renders the correlation function short-ranged with the correlation radius

$$
\lambda_{\mathrm{D}}=\sqrt{\frac{k_{B} T}{4 \pi e^{2} \rho_{0}}} .
$$

As a result, in Eq. (27) the integral over $d \Sigma_{\mu 2}$ is dominated by the region around $x_{1}$ of size $\lambda_{\mathrm{D}}$. Notice that the correlation radius Eq. (28) makes sense only if it is much larger than the mean distance between two nearest electrons, which is order of $\rho_{0}^{-1 / 3}$. This leads to a sufficiently small plasma parameter, i.e., $e^{2} \rho_{0}^{1 / 3} / k_{B} T \ll 1$. Combining with the inequality (24) we obtain

$$
\frac{\hbar^{2} \rho_{0}^{2 / 3}}{m} \ll e^{2} \rho_{0}^{1 / 3} \ll k_{B} T \ll \alpha^{2} m
$$

which is the exact condition for the subsequent analysis to be applicable.

Then, the low density limit introduces a substantial simplification of the collision integral $\mathbb{K}[f]$. Indeed, because the physical one-body distribution is proportional to the density in Eq. (17) the collision integral may be considered formally as a density expansion which, term by term, corresponds to the two-, three-body scattering and so on. In the low density limit all the higher order terms in this density expansion may be ignored. As a result,

$$
\begin{array}{rl}
\mathbb{K}[f]=\int_{\Sigma_{2} \otimes U_{2}^{4}} & d \Sigma_{\mu 2} d^{4} p_{2} u_{2}^{\mu} \\
& \times\langle 1|\mathbf{V}(\boldsymbol{\Gamma}-\hat{\mathfrak{L}}) \mathbf{V}| 1 \mid 2\rangle f(1) f(2) .
\end{array}
$$

Furthermore, with the help of appropriate iteration for Eq. (26) we explicitly write down the operator $\mathbf{V} \boldsymbol{\Gamma V}$ as 34]

$$
\mathbf{V}(\boldsymbol{\Gamma}-\hat{\mathfrak{L}}) \mathbf{V}=\sum_{n=1}^{\infty} \mathbf{V} \boldsymbol{\Gamma}_{[n]} \mathbf{V}
$$

Here

$$
\begin{gathered}
\boldsymbol{\Gamma}_{[n]}=\int_{0}^{\infty} d s_{2} \int_{0}^{\infty} d s_{4} \cdots \int_{0}^{\infty} d s_{2 n} \int_{0}^{s_{1}-s_{2}} d s_{3} \int_{0}^{s_{3}-s_{4}} d s_{5} \cdots \int_{0}^{s_{2 n-3}-s_{2 n-2}} d s_{2 n-1} \mathbf{V} \hat{\mathfrak{g}}\left(s_{2}\right) \mathbf{V} \hat{\mathfrak{U}}\left(s_{1}-s_{2}-s_{3}\right) \mathbf{V} \\
\times \hat{\mathfrak{g}}\left(s_{4}\right) \mathbf{V} \hat{\mathfrak{U}}\left(s_{3}-s_{4}-s_{5}\right) \mathbf{V} \cdots \hat{\mathfrak{g}}\left(s_{2 n}\right) \mathbf{V} \hat{\mathfrak{U}}\left(s_{2 n-1}-s_{2 n}-s_{2 n+1}\right) \mathbf{V},
\end{gathered}
$$

where $s_{1}=s_{2 n+1}=0$, and the operator $\mathbf{V} \hat{\mathfrak{U}}(s) \mathbf{V}=$ $\mathbf{V} \exp \{s \mathbf{V} \hat{\mathfrak{L}} \mathbf{V}\}$. Notice that in order for the matrix element $\left\langle\Gamma_{r}|\mathbf{V} \hat{\mathfrak{g}}(s) \mathbf{V}| \Gamma_{r^{\prime}}^{\prime}\right\rangle$ not to vanish the condition: $\Gamma_{r}=0_{r}, r \geq 2$ and $\Gamma_{r^{\prime}}=0_{r^{\prime}}, r^{\prime}>2$ must be met. For $\boldsymbol{\Gamma}_{[n]}$ with $n \geq 2$ there are more than one particles annihilated, which leads to higher order density corrections as the interaction strength $\lambda$ is compensated by a density factor associated with the annihilated particle. For this reason in the expansion of Eq. (31) only the leading term $(n=1)$ is kept. Furthermore, because in the $\lambda$ expansion of $\mathbf{V} \hat{\mathfrak{U}} \mathbf{V}$ the higher order terms are associated with the particle annihilation resulting in higher-order density corrections, the replacement: $\mathfrak{U}(-s) \rightarrow \hat{\mathfrak{U}}^{0}(-s)$ may be made. Consequently, we obtain

$$
\begin{aligned}
\mathbb{K}[f]= & \int_{\Sigma_{2} \otimes U_{2}^{4}} d \Sigma_{\mu 2} d^{4} p_{2} u_{2}^{\mu} \\
& \times \int_{0}^{\infty} d s\left\langle 1\left|\mathbf{V} \hat{\mathfrak{g}}(s) \mathbf{V} \hat{\mathfrak{U}}^{0}(-s) \mathbf{V}\right| 1 \mid 2\right\rangle f(1) f(2)
\end{aligned}
$$

as the (formal) leading order density expansion of the collision integral (30) concerned.

The simplified collision integral (33) can be expressed in terms of the $\lambda$-expansion. It is important that for this expansion the interaction strength $\lambda$ is not compensated by the density factor because no particles are annihilated. In the remaining part we show that keeping such an expansion up to the $\lambda / \lambda^{2}$ term results in the manifestly covariant Vlasov/Landau equation, while keeping the entire expansion results in the manifestly covariant Boltzmann equation.

Finally let us present a summary of the approximations to be used in the subsequent analysis that implement the scheme outlined above. One is the so-called relativistic impulse approximation [2, 3]. There, the phase trajectory $\mathcal{X}_{(x, p)}$ in Eqs. (17) and (18) is given by

$$
\mathcal{X}_{(x, p)} \equiv x[s]=x^{\mu}-u^{\mu} s
$$

where we choose the proper time origin to be the mo- 
ment at which the world line passes through $x$ with the given 4 -velocity $u^{\mu}$. The other is the traditional hydrodynamic approximation assuming that the physical distribution function $f(x, p)$ varies over a spatial (temporal) scale much larger than $\lambda_{\mathrm{D}}\left(\omega_{p}^{-1}=\sqrt{m / 4 \pi e^{2} \rho_{0}}\right)$. Eq. (33), in combination with these two approximations, is the starting point of subsequent analysis.

\section{B. Mean field approximation: Relativistic Vlasov equation}

Let us start from the simplest case namely to keep the $\lambda$-expansion up to the first order. That is, we neglect the collision integral. Consequently, we obtain

$$
\left\{u_{1}^{\mu} \partial_{\mu 1}-\lambda \int_{\Sigma_{2} \otimes U_{2}^{4}} d \Sigma_{\mu 2} d^{4} p_{2} u_{2}^{\mu} \hat{\mathcal{L}}_{12}^{\prime} f(2)\right\} f(1)=0(35)
$$

According to the second term of Eq. (35), the physical one-body distribution function is driven by the mean field formed by all the other particles. For this reason, to keep the interaction expansion up to the leading order is called mean field approximation. To fully determine the mean field we use the relativistic impulse approximation Eq. (34). As a result,

$$
\begin{gathered}
\lambda \hat{\mathcal{L}}_{12}^{\prime}\left(x_{1}, p_{1} ; x_{2}, p_{2}\right) \approx \frac{i 8 \pi^{2} e^{2}}{m} \int \frac{d^{4} k}{(2 \pi)^{4}} e^{i k \cdot\left(x_{1}-x_{2}\right)} \hat{\mathcal{G}}_{12}(k), \\
\hat{\mathcal{G}}_{12}(k) \equiv \frac{1}{k^{2}}\left\{\delta\left(k \cdot p_{2}\right)\left[k^{\mu}\left(p_{1} \cdot p_{2}\right)-p_{2}^{\mu}\left(k \cdot p_{1}\right)\right] \frac{\partial}{\partial p_{1}^{\mu}}-\delta\left(k \cdot p_{1}\right)\left[k^{\mu}\left(p_{1} \cdot p_{2}\right)-p_{1}^{\mu}\left(k \cdot p_{2}\right)\right] \frac{\partial}{\partial p_{2}^{\mu}}\right\} .
\end{gathered}
$$

The derivation is given in Appendix A2.

\section{Weak coupling approximation: Relativistic Landau equation}

In this part we will consider the so-called weak coupling approximation to Eq. (33). That is, the $\lambda$-expansion is kept up to the second order [Fig. 1 (a)]. The collision integral thereby obtained is denoted as $\mathbb{K}_{1}[f]$.

\section{Collision integral}

Under the weak coupling approximation Eq. (33) is simplified as

$$
\mathbb{K}_{1}[f]=\lambda^{2} \int_{0}^{\infty} d s \int_{\Sigma_{2} \otimes U_{2}^{4}} d \Sigma_{\mu 2} d^{4} p_{2} u_{2}^{\mu} \hat{\mathcal{L}}_{12}^{\prime} \hat{\mathrm{U}}_{12}^{0}(s) \hat{\mathcal{L}}_{12}^{\prime} \hat{\mathrm{U}}_{12}^{0}(-s) f\left(x_{2}, p_{2}\right) f\left(x_{1}, p_{1}\right)
$$

where the propagator $\hat{\mathrm{U}}_{i j}^{0}(s)$ is defined as

$$
\hat{\mathrm{U}}_{i j}^{0}(s)=\exp \left[-s\left(u_{i}^{\mu} \partial_{\mu i}+u_{j}^{\mu} \partial_{\mu j}\right)\right] .
$$

As discussed in Sec. III A because of the short-ranged two-body correlation the integration over $d \Sigma_{\mu 2}$ is dominated by a region of size $\lambda_{\mathrm{D}}$. Applying the hydrodynamic approximation gives

$$
\int_{\Sigma_{2} \otimes U_{2}^{4}} d \Sigma_{\mu 2} d^{4} p_{2} u_{2}^{\mu}(\cdots) f\left(x_{1}, p_{1}\right) f\left(x_{2}, p_{2}\right) \approx \int_{\Sigma_{2} \otimes U_{2}^{4}} d \Sigma_{\mu 2} d^{4} p_{2} u_{2}^{\mu}(\cdots) f\left(x_{1}, p_{1}\right) f\left(x_{1}, p_{2}\right)
$$

for the collision integral $\mathbb{K}_{1}[f]$. That is, the collision integral is local in spacetime:

$$
\mathbb{K}_{1}[f]=\lambda^{2} \int_{0}^{\infty} d s \int_{\Sigma_{2} \otimes U_{2}^{4}} d \Sigma_{\mu 2} d^{4} p_{2} u_{2}^{\mu} \hat{\mathcal{L}}_{12}^{\prime} \hat{\mathrm{U}}_{12}^{0}(s) \hat{\mathcal{L}}_{12}^{\prime} \hat{\mathrm{U}}_{12}^{0}(-s) f\left(x_{1}, p_{2}\right) f\left(x_{1}, p_{1}\right) .
$$

With the relativistic impulse approximation further used we obtain

$$
\begin{aligned}
\mathbb{K}_{1}[f] & =\frac{\partial}{\partial p_{1}^{\mu}} \int d^{4} p_{2} \epsilon^{\mu \nu}\left(\frac{\partial}{\partial p_{1}^{\nu}}-\frac{\partial}{\partial p_{2}^{\nu}}\right) f\left(x_{1}, p_{2}\right) f\left(x_{1}, p_{1}\right), \\
\epsilon^{\mu \nu} & =2 e^{4}\left(u_{1} \cdot u_{2}\right)^{2} \int d^{4} k \delta\left(k \cdot u_{1}\right) \delta\left(k \cdot u_{2}\right) \frac{k^{\mu} k^{\nu}}{(k \cdot k)^{2}}
\end{aligned}
$$


after straightforward but tedious calculations, which are detailed in Appendix B. Eq. (41) is the relativistic Landau collision integral [36]. It was first justified by Klimontovich at the full microscopic level albeit in a nonmanifestly covariant manner [13, 38]. Notice that although the relativistic Landau collision integral is divergent, it formally admits the Jüttner distribution as the unique (local) equilibrium distribution (see Sec. IVA for detailed analysis), and gives the relaxation time (up to a numerical factor) as $\sqrt{m\left(k_{B} T\right)^{3}} /\left(e^{4} \rho_{0}\right) \gg \omega_{p}^{-1}$.

\section{Logarithmic divergence of the collision integral}

However, the collision integral $\mathbb{K}_{1}[f]$ suffers from both the infrared and the ultraviolet divergence. Indeed, with the integral over wave vector carried out Eq. (42) gives (see Appendix C for details)

$$
\begin{aligned}
\epsilon^{\mu \nu}=-2 \pi e^{4} \int \frac{d k_{\perp}}{k_{\perp}} & \left(u_{1} \cdot u_{2}\right)^{2}\left[\left(u_{1} \cdot u_{2}\right)^{2}-1\right]^{-3 / 2} \\
& \times\left\{\left[\left(u_{1} \cdot u_{2}\right)^{2}-1\right] g^{\mu \nu}+\left(u_{1}^{\mu} u_{1}^{\nu}+u_{2}^{\mu} u_{2}^{\nu}\right)-\left(u_{1} \cdot u_{2}\right)\left(u_{1}^{\mu} u_{2}^{\nu}+u_{2}^{\mu} u_{1}^{\nu}\right)\right\} .
\end{aligned}
$$

A similar logarithmic divergence of the collision integral was first noticed by Landau [39] in the context of nonrelativistic plasmas (the so-called Coulomb logarithm). Following the prescription of Landau, from the practical viewpoint, in order to describe nonequilibrium processes near (local) Jüttner equilibrium it suffices to substitute appropriate ultraviolet (infrared) cutoff $k_{\max }\left(k_{\min }\right)$ into the collision integral since the divergence is logarithmic. To further estimate these cutoffs in the relativistic context we notice that $k_{\max }^{-1}$ is the minimal distance as two classical electrons approach each other. At such a distance the kinetic energy and the interaction becomes comparable, i.e.,

$$
k_{\max } \sim \frac{k_{B} T}{e^{2}} .
$$

For moderate temperatures $k_{B} T \ll \alpha^{2} m$ to heal the ultraviolet divergence is well within the reach of the present theory. Physically, the weak coupling approximation namely the leading order $\lambda$-expansion of Eq. (33) accounts for the small angle scattering, but fails in describing large angle scattering. To implement this one needs to sum up the entire $\lambda$-expansion of Eq. (33) or the diagrams shown in Fig. 2. (Let us keep in mind that the collision integral thereby obtained formally is the first order density expansion of $\mathbb{K}[f]$.) This is, indeed, the main issue of Sec. IIID (For higher temperatures the QED scattering processes become dominant, and the complete treatment must be built on a quantum theory.)

The infrared divergence reflects the long-ranged nature of electromagnetic interactions. The present case crucially differs from the nonrelativistic case in that the divergence exists also for the transverse electromagnetic interaction [see Eq. (48), below] which is, however, only dynamically screened [7, 41]. As a result, the infrared divergence has to be healed by the short-ranged correlation, which is to be detailed in Sec. IVB and was first noticed by Klimontovich in the formulation of a nonmanifestly covariant theory [38]. The infrared cutoff is therefore set by inverse correlation radius, i.e.,

$$
k_{\min } \sim \lambda_{\mathrm{D}}^{-1} .
$$

The infrared divergence of the Landau collision integral roots in that the weak coupling approximation fails to capture the collective dielectric effects which leads to the short-ranged two-body correlation. This may be most readily seen by writing the relativistic Landau equation in the observer's frame. For this purpose, let us introduce the distribution function $f_{0}(x, p)$ defined as

$$
f(x, p) \equiv f_{0}(x, p) 2 m \theta\left(p^{0}\right) \delta\left(p^{2}-m^{2}\right) .
$$

To simplify discussions we consider the spatially homogeneous case. [The resulting $f_{0}$ is denoted as $f_{0}(\mathbf{p}, t)$.] Then, the kinetic equation (17) with the collision integral $\mathbb{K}_{1}[f]$ can be exactly rewritten as $[38]$

$\frac{\partial}{\partial t} f_{0}(\mathbf{p}, t)=\frac{\partial}{\partial \mathbf{p}} \cdot \int d \mathbf{p}^{\prime} \mathbf{Q} \cdot\left(\frac{\partial}{\partial \mathbf{p}}-\frac{\partial}{\partial \mathbf{p}^{\prime}}\right) f_{0}(\mathbf{p}, t) f_{0}\left(\mathbf{p}^{\prime}, t\right)$.

Here the tensor (in the 3-dimensional Euclidean space) $\mathbf{Q}$ reads out as ( $\mathbf{v}$ and $\mathbf{v}^{\prime}$ below are the velocity.)

$$
\begin{aligned}
& \mathbf{Q}=2 e^{4} \int d \omega \int d^{3} \mathbf{k} \delta(\omega-\mathbf{k} \cdot \mathbf{v}) \delta\left(\omega-\mathbf{k} \cdot \mathbf{v}^{\prime}\right) \\
& \times \frac{\mathbf{k k}}{|\mathbf{k}|^{4}}\left\{\frac{1}{\left|\varepsilon^{\|}(\omega, \mathbf{k})\right|^{2}}+\frac{\left[(\mathbf{k} \times \mathbf{v}) \cdot\left(\mathbf{k} \times \mathbf{v}^{\prime}\right)\right]^{2}}{\left|\omega^{2} \varepsilon^{\perp}(\omega, \mathbf{k})-\mathbf{k}^{2}\right|^{2}}\right\},
\end{aligned}
$$

where $\varepsilon^{\|}(\omega, \mathbf{k})=\varepsilon^{\perp}(\omega, \mathbf{k})=1$. The second term in the curl bracket arises from the interaction mediated by the transverse electromagnetic field. It is negligibly small in the nonrelativistic limit, i.e., $|\mathbf{v}|,\left|\mathbf{v}^{\prime}\right| \ll 1$, while the first term arises from the interaction mediated by the longitudinal electromagnetic field and possesses the general structure of the Landau-Balescu-Lenard equation [20, 39]. In comparing with the nonmanifestly kinetic equation obtained by Klimontivich [38] Eq. (48) suggests that the longitudinal (transverse) permittivity $\varepsilon^{\|}\left(\varepsilon^{\perp}\right)$ is 


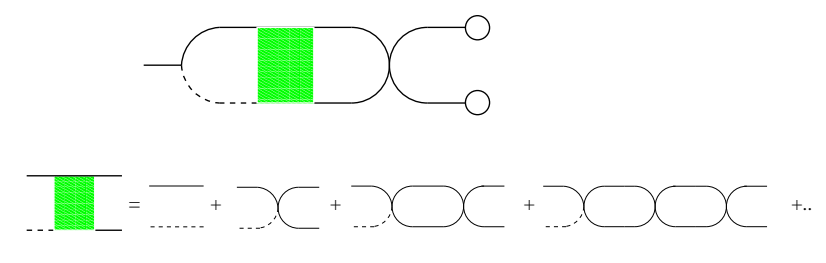

FIG. 2: Diagrams leading to the Boltzmann collision integral.

unity and, thus, the collective dielectric response is completely ignored. Nevertheless this is an artifact of the weak coupling approximation. In fact, the nonmanifestly covariant theory [38] has shown

$$
\begin{aligned}
& \varepsilon^{\|}(\omega \rightarrow 0, \mathbf{k})-1 \propto \frac{1}{\left(|\mathbf{k}| \lambda_{\mathrm{D}}\right)^{2}}, \\
& \varepsilon^{\perp}(\omega \rightarrow 0, \mathbf{k})-1 \propto \frac{i e^{2}}{\omega|\mathbf{k}|},
\end{aligned}
$$

which suggests that the fluctuations of the longitudinal (transverse) electromagnetic field acquire a static (dynamical) mass $\propto e^{2}$ (namely the interaction strength). By inserting Eq. (49) into Eq. (48) it is clear that for a collision integral to account for the collective dielectric effects to go beyond the weak coupling approximation is inevitable. More precisely, (in the Newtonian physics) the collective dielectric effect is well known to result in a short-ranged two-body correlation, and to be responsible for by more complicated correlation associated with higher order terms in the density expansion of the collision integral [20].

In the present context to treat the infrared divergence accurately we need to sum up all the so-called ring $d i$ agrams (to be defined in Sec. IVB). In Fig. 1 (b) we present the lowest order ring diagrams. In doing so we expect to obtain a manifestly covariant generalization of the Balescu-Lenard equation, where the infrared divergence is healed by collective dielectric effects. However, to carry out this program is far beyond the scope of the present work. We here limit ourselves to confirm this important observation in the case of global equilibrium, which is detailed in Sec. IVB.

\section{Two-body scattering approximation: Relativistic Boltzmann equation}

In this part we wish to justify the relativistic Boltzmann equation. The derivation below may be generalized to low density systems with short-ranged interactions, where the two-body scattering dominates.

\section{Formal collision integral}

We first introduce the following propagator:

$$
\hat{\mathcal{U}}_{i j z}=\sum_{n=0}^{\infty} \lambda^{n}\left\langle i j\left|\mathbf{C} \hat{\mathfrak{R}}^{0}(z)\left[\hat{\mathfrak{L}}^{\prime} \mathbf{C} \hat{\mathfrak{R}}^{0}(z)\right]^{n}\right| i j\right\rangle .
$$

Upon passing to the time representation: $\hat{\mathcal{U}}_{i j z} \rightarrow \hat{\mathcal{U}}_{i j}(s)$, we find

$$
\frac{\partial}{\partial s} \hat{\mathcal{U}}_{i j}(s)=\left(\hat{\mathcal{L}}_{i}^{0}+\hat{\mathcal{L}}_{j}^{0}+\lambda \hat{\mathcal{L}}_{i j}^{\prime}\right) \hat{\mathcal{U}}_{i j}(s), \hat{\mathcal{U}}_{i j}(0)=1 .
$$

Then, for Eq. (33) we keep the entire $\lambda$-expansion (Fig. 2) which is called the two-body scattering approximation. The collision integral thereby obtained is denoted as $\mathbb{K}_{2}[f]$ which reads out as

$$
\begin{aligned}
\mathbb{K}_{2}[f]= & \lambda^{2} \int_{0}^{\infty} d s \int_{\Sigma_{2} \otimes U_{2}^{4}} d \Sigma_{\mu 2} d^{4} p_{2} u_{2}^{\mu} \\
& \times \hat{\mathcal{L}}_{12}^{\prime} \hat{\mathcal{U}}_{12}(s) \hat{\mathcal{L}}_{12}^{\prime} \hat{\mathrm{U}}_{12}^{0}(-s) f\left(x_{2}, p_{2}\right) f\left(x_{1}, p_{1}\right) .
\end{aligned}
$$

It differs from Eq. (40) in that the propagator $\hat{\mathrm{U}}_{12}^{0}(s)$ sandwiched by $\hat{\mathcal{L}}_{12}^{\prime}$ is renormalized into $\hat{\mathcal{U}}_{12}(s)$ (Fig. 2 , lower panel).

The derivation is exact so far. In the leading order hydrodynamic expansion the spacetime inhomogeneity of $f(x, p)$ and two-body scattering are decoupled. That is, we may also apply Eq. (39) to the collision integral $\mathbb{K}_{2}[f]$ and, furthermore, approximate the operator $\hat{\mathrm{U}}_{12}^{0}(-s)$ in $\mathbb{K}_{2}[f]$ by unity. As a result, with $s$ integrated out Eq. (52) is simplified as

$$
\begin{array}{rl}
\mathbb{K}_{2}[f]=\lambda^{2} \int_{\Sigma_{2} \otimes U_{2}^{4}} & d \Sigma_{\mu 2} d^{4} p_{2} u_{2}^{\mu} \\
\times \hat{\mathcal{L}}_{12}^{\prime} \hat{Z} \hat{\mathcal{L}}_{12}^{\prime} f\left(x_{1}, p_{2}\right) f\left(x_{1}, p_{1}\right)
\end{array}
$$

where $\hat{Z}=\int_{0}^{\infty} d s \hat{\mathcal{U}}_{12}(s)$. In order to find $\hat{Z}$ we use Eq. (51) to set up the following equation:

$$
\hat{Z}=\hat{G}+\hat{G} \lambda \hat{\mathcal{L}}_{12}^{\prime} \hat{Z}, \quad \hat{G}=\int_{0}^{\infty} d s \hat{\mathrm{U}}_{12}^{0}(s) .
$$

\section{Two-body scattering and collision integral}

As before we employ the relativistic impulse approximation. There we insert Eq. (34) into the two-body interacting Liouvillian $\lambda \hat{\mathcal{L}}_{12}^{\prime}$. To proceed further we notice that to describe the free motion of particles 1 and 2 , with the 4 -momentum vector $p_{1}$ and $p_{2}$, respectively, we may work in the center-of-momentum (CM) frame (Correspondingly we use the prime to denote vectors in this frame.) where $\mathbf{p}_{1}^{\prime}+\mathbf{p}_{2}^{\prime}=0$. Because the correlation radius is short-ranged they do not interact with each other until at a distance of $\sim \lambda_{\mathrm{D}}$, when they start to repel each other. Eventually as their deviation reaches 
order of $\lambda_{\mathrm{D}}$ they undergo free flight again. Then, we assume that in the CM frame the interaction between two particles is switched on simultaneously. This, indeed, is perfectly legitimate because the hydrodynamic approximation washes out effects arising from the small (coordinate) time mismatch $\sim \lambda_{\mathrm{D}}$ of two particles. (Such an assumption may be released if we simplify short-ranged interactions as point-like collisions. In the latter case it can be shown that the derivations below are exact.) As such we find that, in the CM frame,

$$
\mathbf{p}_{1}^{\prime}\left(t^{\prime}\right)+\mathbf{p}_{2}^{\prime}\left(t^{\prime}\right)=0
$$

for all $t^{\prime}$, moreover, the two-body interacting Liouvillian does not depend on the coordinate time $t^{\prime}$ any more:

$$
\lambda \hat{\mathcal{L}}_{i j}^{\prime}\left(x_{i}^{\prime}, p_{i}^{\prime} ; x_{j}^{\prime}, p_{j}^{\prime}\right) \equiv \lambda \hat{\mathcal{L}}_{i j}^{\prime}\left(\mathbf{x}_{i}^{\prime}-\mathbf{x}_{j}^{\prime}, p_{i}^{\prime}, p_{j}^{\prime}\right) .
$$

That is, in such frame the two-body dynamics may be reduced into the dynamics of single particle subject to some external time-independent potential. The detailed analysis is presented in Appendix A3.

Let us introduce the following notations: $u^{\prime}=p_{1}^{\prime} / m \equiv$ $\left(u^{\prime 0}, \mathbf{u}^{\prime}\right), r^{\prime} \equiv\left(t^{\prime}, x^{\prime}, y^{\prime}, z^{\prime}\right) \equiv\left(t_{1}^{\prime}=t_{2}^{\prime},\left(\mathbf{x}_{1}^{\prime}-\mathbf{x}_{2}^{\prime}\right) / 2\right)$ assuming that the particle 1 approaches to (departures from) particle 2 from $z^{\prime}=-\infty\left(\right.$ at $\left.z^{\prime}=+\infty\right)$. Since $\lambda \hat{\mathcal{L}}_{12}^{\prime}$ is time-independent in the CM frame, from Eq. (53) we see that in the leading order hydrodynamic expansion the derivative with respect to $t^{\prime}$ involved in the definition of $\hat{G}$, namely Eq. (54) may be dropped out. As a result, the matrix element of $\hat{G}$, denoted as $G\left(\mathbf{r}^{\prime}, p^{\prime} ; \tilde{\mathbf{r}}^{\prime}, \tilde{p}^{\prime}\right)$ satisfies

$$
2 \mathbf{u}^{\prime} \cdot \frac{\partial}{\partial \mathbf{r}^{\prime}} G\left(\mathbf{r}^{\prime}, p^{\prime} ; \tilde{\mathbf{r}}^{\prime}, \tilde{p}^{\prime}\right)=\delta^{(3)}\left(\mathbf{r}^{\prime}-\tilde{\mathbf{r}}^{\prime}\right) \delta^{(4)}\left(p^{\prime}-\tilde{p}^{\prime}\right) .
$$

Using this equation it can be checked that the matrix element of $\hat{Z}$, denoted as $Z\left(\mathbf{r}^{\prime}, p^{\prime} ; \tilde{\mathbf{r}}^{\prime}, \tilde{p}^{\prime}\right)$ solves

$$
\begin{gathered}
\left\{2 \mathbf{u}^{\prime} \cdot \frac{\partial}{\partial \mathbf{r}^{\prime}}-\lambda \hat{\mathcal{L}}_{12}^{\prime}\right\} Z\left(\mathbf{r}^{\prime}, p^{\prime} ; \tilde{\mathbf{r}}^{\prime}, \tilde{p}^{\prime}\right) \\
=\delta^{(3)}\left(\mathbf{r}^{\prime}-\tilde{\mathbf{r}}^{\prime}\right) \delta^{(4)}\left(p^{\prime}-\tilde{p}^{\prime}\right) .
\end{gathered}
$$

Analogous to quantum mechanics Eq. (58) describe the scattering of an incident "wave function" $\psi_{\text {in }}\left(p^{\prime}\right)$ under the "potential" which now reads out as $-\lambda \hat{\mathcal{L}}_{12}^{\prime}$. And the out-going "wave function" $\psi_{\text {out }}\left(\mathbf{r}^{\prime}, p^{\prime}\right)$ is

$$
\begin{aligned}
& \psi_{\text {out }}\left(\mathbf{r}^{\prime}, p^{\prime}\right)=\psi_{\text {in }}\left(p^{\prime}\right) \\
& \quad+\lambda \int d^{3} \tilde{\mathbf{r}}^{\prime} \int_{U^{4}} d^{4} \tilde{p}^{\prime} \tilde{u}^{\prime 0} Z\left(\mathbf{r}^{\prime}, p^{\prime} ; \tilde{\mathbf{r}}^{\prime}, \tilde{p}^{\prime}\right) \hat{\mathcal{L}}_{12}^{\prime} \psi_{\text {in }}\left(\tilde{p}^{\prime}\right) .
\end{aligned}
$$

Using Eq. (58) we find

$$
\left\{2 \mathbf{u}^{\prime} \cdot \frac{\partial}{\partial \mathbf{r}^{\prime}}-\lambda \hat{\mathcal{L}}_{12}^{\prime}\right\} \psi_{\text {out }}\left(\mathbf{r}^{\prime}, p^{\prime}\right)=0
$$

In deriving this equation we keep in mind that the twobody scattering is a local event with a characteristic scale $\lambda_{\mathrm{D}}$. Over such a scale incident wave functions are strongly scattered (by the potential $-\lambda \hat{\mathcal{L}}_{12}^{\prime}$ ) and, as such, the out-going wave function generally acquires a strong dependence on $\mathbf{r}^{\prime}$. In contrast, as the two-body scattering (in the CM frame) concerned the incident wave function $\psi_{\text {in }}$ in Eq. (59) may be regarded as a spacetime independent object. Nevertheless we notice that this is not true in the observer's frame where, instead, the incident wave function reads out as

$$
\psi_{\text {in }}\left(p^{\prime}\right)=f\left(x_{1}, p_{1}\right) f\left(x_{1}, p_{2}\right)
$$

varying over a macroscopic scale $\gtrsim \lambda_{\mathrm{D}}$. In Eq. (61) $p^{\prime}$ is uniquely determined by $p_{1,2}$.

Substituting Eq. (59) into Eq. (53) we find that, in terms of the CM frame coordinates, the collision integral is

$$
\begin{aligned}
\mathbb{K}_{2}[f] & =\lambda \int d^{3} \mathbf{r}^{\prime} \int_{U^{4}} d^{4} p^{\prime} u^{\prime 0} \hat{\mathcal{L}}_{12}^{\prime}\left\{\psi_{\text {out }}\left(\mathbf{r}^{\prime}, p^{\prime}\right)-\psi_{\text {in }}\left(p^{\prime}\right)\right\} \\
& =\lambda \int d^{3} \mathbf{r}^{\prime} \int_{U^{4}} d^{4} p^{\prime} u^{\prime 0} \hat{\mathcal{L}}_{12}^{\prime} \psi_{\text {out }}\left(\mathbf{r}^{\prime}, p^{\prime}\right) \\
& =\int_{U^{4}} d^{4} p^{\prime} \int d^{3} \mathbf{r}^{\prime} 2 u^{\prime 0} \mathbf{u}^{\prime} \cdot \frac{\partial}{\partial \mathbf{r}^{\prime}} \psi_{\text {out }}\left(\mathbf{r}^{\prime}, p^{\prime}\right)
\end{aligned}
$$

where in deriving the second line we use the fact that $\int d^{3} \mathbf{r}^{\prime} \hat{\mathcal{L}}_{12}^{\prime}=0$, and in deriving the third line we use Eq. (60).

\section{Boltzmann collision integral}

For Eq. (62) let us integrate out $\mathbf{r}^{\prime}$ first. For this purpose we fix the $z^{\prime}$-axis to be in the direction of $\mathbf{u}^{\prime}$. Then,

$$
\begin{aligned}
\int d^{3} \mathbf{r}^{\prime} 2 u^{\prime 0} \mathbf{u}^{\prime} & \cdot \frac{\partial}{\partial \mathbf{r}^{\prime}} \psi_{\text {out }}\left(\mathbf{r}^{\prime}, p^{\prime}\right)=\int d^{3} \mathbf{r}^{\prime} 2 u^{\prime 0}\left|\mathbf{u}^{\prime}\right| \frac{\partial}{\partial z^{\prime}} \psi_{\text {out }}\left(\mathbf{r}^{\prime}, p^{\prime}\right) \\
& =\int d x^{\prime} d y^{\prime}\left\{u^{\prime 0}\left|\mathbf{u}^{\prime}\right|-u^{\prime 0}\left(-\left|\mathbf{u}^{\prime}\right|\right)\right\}\left\{\left.\psi_{\text {out }}\left(\mathbf{r}^{\prime}, p^{\prime}\right)\right|_{z^{\prime} \rightarrow+\infty}-\left.\psi_{\text {out }}\left(\mathbf{r}^{\prime}, p^{\prime}\right)\right|_{z^{\prime} \rightarrow-\infty}\right\}
\end{aligned}
$$


Remarkably, Eq. (63) depends only on the wave functions at $z^{\prime}= \pm \infty$ and is irrespective of the details of the twobody scattering. Noticing that the interaction vanishes at $z^{\prime} \rightarrow \pm \infty$ we thus find $\left.\psi_{\text {out }}\left(\mathbf{r}^{\prime}, p^{\prime}\right)\right|_{z^{\prime} \rightarrow-\infty}=\psi_{\text {in }}\left(p^{\prime}\right)$. In the CM frame applying the Liouville theorem further gives

$$
\begin{aligned}
\left.\psi_{\text {out }}\left(\mathbf{r}^{\prime}, p^{\prime}\right)\right|_{z^{\prime} \rightarrow+\infty} & =\psi_{\text {in }}\left(\tilde{p}^{\prime}\left(\Omega^{\prime}\right)\right) \\
& =f\left(x_{1}, \tilde{p}_{1}\right) f\left(x_{1}, \tilde{p}_{2}\right) .
\end{aligned}
$$

Here $\tilde{p}^{\prime}\left(\Omega^{\prime}\right)$ is the out-going 4-momentum vector depending on the scattering angle $\Omega^{\prime}$. In deriving the second equality we use Eq. (61) and pass to the observer's frame with $\tilde{p}_{1,2}$ standing for the out-going 4-momentum vector of the two particles. To proceed further we introduce the differential cross section in the CM frame $\sigma_{\mathrm{cm}}$ defined as $d x^{\prime} d y^{\prime} \equiv \sigma_{\mathrm{cm}}\left(p_{1}, p_{2} \rightarrow \tilde{p}_{1}, \tilde{p}_{2}\right) d \Omega^{\prime}$. By definition, $\sigma_{\mathrm{cm}}$ is a manifestly covariant concept. Moreover, in the observer's frame the quantity $u^{\prime 0}\left|\mathbf{u}^{\prime}\right|-u^{\prime 0}\left(-\left|\mathbf{u}^{\prime}\right|\right)$ may be written as $\sqrt{\left(u_{1} \cdot u_{2}\right)^{2}-1}$. Collecting everything together and taking into account Eq. (46), we obtain

$$
\begin{aligned}
& \left\{u_{1}^{\mu} \partial_{\mu 1}-\int_{\Sigma_{2} \otimes U_{2}^{4}} d \Sigma_{\mu 2} d^{4} p_{2} u_{2}^{\mu} \lambda \hat{\mathcal{L}}_{12}^{\prime} f_{0}(2)\right\} f_{0}(1) \\
= & \mathbb{K}_{2}\left[f_{0}\right] .
\end{aligned}
$$

Here the collision integral is

$$
\begin{aligned}
\mathbb{K}_{2}[f]= & \iint d \Omega^{\prime} \frac{d^{3} p_{2}}{p_{2}^{0}} \sigma_{\mathrm{cm}} \sqrt{\left(u_{1} \cdot u_{2}\right)^{2}-1} \\
& \times\left\{f_{0}\left(x_{1}, \tilde{p}_{1}\right) f_{0}\left(x_{1}, \tilde{p}_{2}\right)-f_{0}\left(x_{1}, p_{1}\right) f_{0}\left(x_{1}, p_{2}\right)\right\} .
\end{aligned}
$$

Eq. (66) is the well known relativistic Boltzmann collision integral [10, 11], where the details of the two-body scattering enter through the invariant differential cross section $\sigma_{\mathrm{cm}}$.

\section{CORRELATION AT RELATIVISTIC MANY-BODY EQUILIBRIUM}

It is important that the perceptions of a macroscopic observer correspond to the hierarchy of physical correlation functions namely $\overrightarrow{\mathfrak{N}}_{\infty}$ rather than $\overrightarrow{\mathfrak{D}}$, and the hierarchy is fully determined by the solution to the general transport equation (17) with the collision integral given by Eq. (18). Obviously, the processes with regard to the physical distribution described by Eq. (17) are observerindependent which, in the chosen inertial frame, may be parametrized by the coordinate time. For rarefied plasmas Eq. (17) becomes a local equation, and the collision integral generally admits the so-called "collision invariants" which, typically, are $\phi(p) \equiv 1, p^{\mu}$, i.e.,

$$
\int_{U^{4}} d^{4} p \phi(p) \mathbb{K}[f(x, p)]=0 .
$$

They amount to the particle number and the energymomentum conservation law, from which macroscopic relativistic hydrodynamics follows.

As the system reaches global or local equilibrium the physical one-body distribution function nullifies the collision integral, i.e., $\mathbb{K}\left[f_{\text {eq }}\right]=0$. The former differs from the latter in the spacetime independence of $f_{\text {eq }}$. By inserting $f_{\text {eq }}$ into Eq. (19) we obtain

$$
\begin{aligned}
\left\langle\Gamma_{j}(1, \cdots, j)\right| \overrightarrow{\mathfrak{N}}_{\infty}=\int_{0}^{\infty} d s \int_{0}^{s} d s^{\prime} & \sum_{n=j}^{\infty} \prod_{i=j+1}^{n} \int_{\Sigma_{i} \otimes U_{i}^{4}} d \Sigma_{\mu i} d^{4} p_{i} u_{i}^{\mu} \prod_{k=1}^{n} f_{\mathrm{eq}}\left(x_{k}, p_{k}\right) \\
& \times\left\langle\Gamma_{j}(1, \cdots, j)\left|\mathbf{C} \hat{\mathfrak{U}}^{0}\left(s-s^{\prime}\right) \hat{\mathfrak{E}}\left(s^{\prime}\right) \mathbf{V} \exp (-s \mathbf{V} \boldsymbol{\Gamma V})\right| 1|\cdots| n\right\rangle
\end{aligned}
$$

which, in principle, gives the entire hierarchy of physical equilibrium correlation functions. Therefore, the conjecture of Hakim on the many-body equilibrium [9] is justified. In Ref. [9] only the first component, i.e., $\langle 12| \overrightarrow{\mathfrak{N}}_{\infty}$ is formulated using the weak coupling approximation. This hierarchy, together with $f_{\text {eq }}(x, p)$ fully captures the many-body equilibrium of rarified relativistic plasmas. It allows one to go beyond the kinetic approximation [2, 3] to formulate the relativistic many-body equilibrium basing on the action-at-a-distance formalism.

\section{A. Jüttner equilibrium}

Let us now exemplify these general principles in the case of $\mathbb{K}_{1,2}[f]$, which can be easily shown to preserve Eq. (67). This then results in the relativistic hydrodynamics-a well established subject [11] which we shall not proceed further. Encompassed by the hydrodynamics the system irreversibly evolves into the local Jüttner equilibrium. Indeed, define the local entropy flux 
$S^{\mu}(x)$ as follows:

$S^{\mu}(x) \equiv-\int d^{4} p 2 m \theta\left(p^{0}\right) \delta\left(p^{2}-m^{2}\right) u^{\mu} f_{0}(x, p) \ln f_{0}(x, p)$,

where $f_{0}(x, p)$ is defined by Eq. (46). Then, from the collision integral (66) the $H$-theorem follows [11], i.e.,

$$
\sigma_{s}(x) \equiv \partial_{\mu} S^{\mu}(x) \geq 0
$$

The local entropy production $\sigma_{s}(x)$ vanishes wherever $f_{0}(x, p)$ reaches the local Jüttner equilibrium:

$$
f_{J}(x, p)=\frac{\rho(x) \beta(x)}{4 \pi m^{2} K_{2}(m \beta(x))} e^{-\beta^{\mu}(x) p_{\mu}} .
$$

Here $\rho(x)$ is the invariant particle number density, $K_{2}$ is the modified Bessel function of order two, and $\beta^{\mu}(x)$ is the (local) reciprocal temperature timelike 4 -vector defining the (local) temperature $T(x)$ through $1 / k_{B} T(x) \equiv$ $\sqrt{\beta(x)^{2}}$. Suppressing all the spacetime dependence of the parameters of $f_{J}(x, p)$ further leads to the (global) Jüttner equilibrium:

$$
f_{\mathrm{eq}}(p) \equiv f_{J}(p) 2 m \theta\left(p^{0}\right) \delta\left(p^{2}-m^{2}\right) .
$$

The debate on the special relativistic version of the Maxwell-Boltzmann distribution has stemmed from Ref. [17] where a different Boltzmann equation, though manifestly covariant, is proposed. There, proceeding along the line similar to Eqs. (69)-(71) results in an alternative one-body equilibrium distribution. In the present work by the proof of the relativistic Landau and Boltzmann equation namely Eq. (17) with the collision integral (41) and (66) is implied the following important fact: In rarefied plasmas the Jüttner distribution is no longer a phenomenological hypothesis, rather, is well justified at the full microscopic level and, importantly, suits the covariant principle. Thus, we provide a solid support for very recent 1-dimensional numerical simulation [26] and advocates the critical analysis on alternatives to the Jüttner distribution [27]. Notice that the spatial dimensionality in the numerical simulation differs which, we believe, is of minor importance. (In fact, for plasmas the relativistic many-body dynamics and thereby the collision integral are substantially simplified in the 1-dimensional geometry. There, the electromagnetic interaction is purely longitudinal.) What is crucial is that, there, the underlying many-body dynamics is dictated by point-like collisions and is well described by the actionat-a-distance formalism and, as a result, the entire scope of the present manifestly covariant correlation dynamics applies.

\section{B. Equal-time two-body correlation}

We further proceed to calculate the correlation function at the Jüttner equilibrium. For simplicity let us consider the simplest two-body correlation function, which is $C_{\text {eq }}\left(x_{1}, p_{1} ; x_{2}, p_{2}\right) \equiv\langle 12| \overrightarrow{\mathfrak{N}}_{\infty}$. Here we use the subscript "eq" to denote that the physical one-body distribution is at global Jüttner equilibrium, i.e., $f\left(x_{i}, p_{i}\right)=f_{\text {eq }}\left(p_{i}\right)$. In particular, to investigate the behavior of the spacelike correlation function we will calculate below the so-called equal-time correlation function.

\section{Bare correlation function}

To the lowest order interaction expansion [see Fig. 3 (a)] Eq. (68) gives a bare two-body correlation function:

$$
\begin{aligned}
& C_{\mathrm{eq}}^{0}\left(x_{1}, p_{1} ; x_{2}, p_{2}\right) \\
= & \int_{0}^{\infty} d s\left\langle 12\left|\hat{\mathfrak{U}}^{0}(s) \lambda \hat{\mathfrak{L}}^{\prime}\right| 1 \mid 2\right\rangle f_{\mathrm{eq}}\left(p_{1}\right) f_{\mathrm{eq}}\left(p_{2}\right) .
\end{aligned}
$$

Under the relativistic impulse approximation (see Appendix A2 for details) it is translationally invariant, i.e., $C_{\text {eq }}\left(x_{1}, p_{1} ; x_{2}, p_{2}\right) \equiv C_{\text {eq }}^{0}\left(x_{1}-x_{2}, p_{1}, p_{2}\right)$. Indeed, inserting the matrix elements of $\hat{\mathfrak{U}}_{0}$ and $\lambda \hat{\mathfrak{L}}^{\prime}$ into Eq. (173) we find

$$
C_{\text {eq }}^{0}\left(x_{1}-x_{2}, p_{1}, p_{2}\right)=\int \frac{d^{4} k}{(2 \pi)^{4}} e^{i k \cdot\left(x_{1}-x_{2}\right)} \tilde{C}_{\mathrm{eq}}^{0}\left(k, p_{1}, p_{2}\right)
$$

with

$$
\begin{aligned}
\tilde{C}_{\mathrm{eq}}^{0}\left(k, p_{1}, p_{2}\right)=-\frac{i 8 \pi^{2} e^{2}}{k^{2}} \frac{1}{i k \cdot\left(p_{1}-p_{2}\right)}\left\{\delta\left(k \cdot p_{2}\right)\left[k \cdot \beta\left(p_{1} \cdot p_{2}\right)-\beta \cdot p_{2}\left(k \cdot p_{1}\right)\right]\right. \\
\left.-\delta\left(k \cdot p_{1}\right)\left[k \cdot \beta\left(p_{1} \cdot p_{2}\right)-\beta \cdot p_{1}\left(k \cdot p_{2}\right)\right]\right\} f_{\mathrm{eq}}\left(p_{1}\right) f_{\mathrm{eq}}\left(p_{2}\right),
\end{aligned}
$$

where the wave vector $k^{\mu} \equiv(\omega, \mathbf{k})$.

Since the equilibrium is global we may take the advantage of the Lorentz invariance of Eq. (74) and choose the observer's frame where $\beta^{\mu}=\left(1 / k_{B} T, 0\right)$, and the spacelike 3 -surface is the usual 3-dimensional Euclidean space. In 
this frame the bare equal-time correlation function is

$$
\begin{aligned}
C_{\mathrm{eq}}^{0}\left(\mathbf{x}_{1}-\mathbf{x}_{2}, p_{1}, p_{2}\right) & =\int \frac{d \mathbf{k}}{(2 \pi)^{3}} e^{i \mathbf{k} \cdot\left(\mathbf{x}_{1}-\mathbf{x}_{2}\right)} \mathcal{C}_{\mathrm{eq}}^{0}\left(\mathbf{k}, p_{1}, p_{2}\right), \\
\mathcal{C}_{\mathrm{eq}}^{0}\left(\mathbf{k}, p_{1}, p_{2}\right) & =\int \frac{d \omega}{2 \pi} \tilde{C}_{\mathrm{eq}}^{0}\left(\omega, \mathbf{k}, p_{1}, p_{2}\right) .
\end{aligned}
$$

Notice that in the observer's frame Eq. (75) is written as

$$
\begin{aligned}
\tilde{C}_{\mathrm{eq}}^{0}\left(\omega, \mathbf{k}, p_{1}, p_{2}\right)=-\frac{i 8 \pi^{2} e^{2}}{k^{2}}\left\{\delta\left(k \cdot p_{2}\right)\left[\frac{\omega}{k_{B} T} \frac{\left(p_{1} \cdot p_{2}\right)}{i\left(\omega p_{1}^{0}-\mathbf{k} \cdot \mathbf{p}_{1}\right)}+\frac{i p_{2}^{0}}{k_{B} T}\right]\right. \\
\left.+\delta\left(k \cdot p_{1}\right)\left[\frac{\omega}{k_{B} T} \frac{\left(p_{1} \cdot p_{2}\right)}{i\left(\omega p_{2}^{0}-\mathbf{k} \cdot \mathbf{p}_{2}\right)}+\frac{i p_{1}^{0}}{k_{B} T}\right]\right\} f_{\mathrm{eq}}\left(p_{1}\right) f_{\mathrm{eq}}\left(p_{2}\right) .
\end{aligned}
$$

Integrating out $\omega$ we find

$$
\mathcal{C}_{\text {eq }}^{0}\left(\mathbf{k}, p_{1}, p_{2}\right) \propto-\frac{f_{\text {eq }}\left(p_{1}\right) f_{\text {eq }}\left(p_{2}\right)}{|\mathbf{k}|^{2}},
$$

where the irrelevant numerical overall factor is ignored. Eq. (78) shows that the bare equal-time two-body correlation is long-ranged, i.e., $C_{\text {eq }}^{0}\left(\mathbf{x}_{1}-\mathbf{x}_{2}, p_{1}, p_{2}\right) \propto\left|\mathbf{x}_{1}-\mathbf{x}_{2}\right|^{-1}$ reflecting the long-ranged nature of electromagnetic interactions.

\section{Short-ranged equal-time correlation: Collective dielectric effects}

To go beyond the weak coupling approximation we extend the so-called ring approximation [34] to the relativistic context. That is, we will sum up all the so-called ring diagrams. [Typical diagrams are given in Fig. 3] (b)-(d).] They are obtained from Fig. 3 (a) in the following way: The propagating lines to the left of the vertex-associated with no particle annihilation-are dressed by a sequence of vertexes. For each of them an additional particle joins the vertex from the right and continues propagating to the left, and eventually is annihilated at the next vertex. (It can be shown that the propagating lines to the right suffer no renormalization effects.) In selecting these diagrams it is implied that in Eq. (68) $\exp (-s \mathbf{V} \boldsymbol{\Gamma V})$ is set to be unity. Summing up all these diagrams we find that the two-body correlation function solves the following Dyson equation:

$$
\begin{aligned}
C_{\text {eq }}\left(x_{1}, p_{1} ; x_{2}, p_{2}\right)=C_{\text {eq }}^{0}\left(x_{1}, p_{1} ; x_{2}, p_{2}\right) & +\int_{0}^{\infty} d s e^{-s\left(u_{1}^{\mu} \partial_{\mu 1}+u_{2}^{\mu} \partial_{\mu 2}\right)} \int_{\Sigma_{3} \otimes U_{3}^{4}} d \Sigma_{\mu 3} d^{4} p_{3} u_{3}^{\mu} \\
& \times\left\{\lambda \hat{\mathcal{L}}_{13}^{\prime} f_{\text {eq }}\left(p_{1}\right) C_{\text {eq }}\left(x_{3}, p_{3} ; x_{2}, p_{2}\right)+\lambda \hat{\mathcal{L}}_{23}^{\prime} f_{\text {eq }}\left(p_{2}\right) C_{\text {eq }}\left(x_{3}, p_{3} ; x_{1}, p_{1}\right)\right\} .
\end{aligned}
$$

Let us now pass to the observer's frame chosen above, where $t_{1}=t_{2}=t_{3}$ and calculate the equal-time correlation using Eq. (79). At global equilibrium such a correlation function does not depend on the coordinate time. Consequently, upon passing to the spatial Fourier transformation we obtain

$$
\begin{aligned}
\mathcal{C}_{\mathrm{eq}}\left(\mathbf{k}, p_{1}, p_{2}\right)= & \mathcal{C}_{\mathrm{eq}}^{0}\left(\mathbf{k}, p_{1}, p_{2}\right)+i 8 \pi^{2} e^{2} \int \frac{d \omega}{2 \pi} \frac{1}{\omega^{2}-|\mathbf{k}|^{2}} \int d^{4} p_{3} u_{3}^{0} \frac{\delta\left(k \cdot p_{3}\right)}{i \mathbf{k} \cdot\left(\mathbf{p}_{1}-\mathbf{p}_{2}\right)} \\
& \times\left\{\left[\frac{\omega}{k_{B} T}\left(p_{1} \cdot p_{3}\right)-\frac{p_{3}^{0}}{k_{B} T}\left(k \cdot p_{1}\right)\right] f_{\mathrm{eq}}\left(p_{1}\right) \mathcal{C}_{\mathrm{eq}}\left(\mathbf{k}, p_{3}, p_{2}\right)\right. \\
& \left.-\left[\frac{\omega}{k_{B} T}\left(p_{2} \cdot p_{3}\right)-\frac{p_{3}^{0}}{k_{B} T}\left(k \cdot p_{2}\right)\right] f_{\mathrm{eq}}\left(p_{2}\right) \mathcal{C}_{\mathrm{eq}}\left(\mathbf{k}, p_{3}, p_{1}\right)\right\},
\end{aligned}
$$

where $\mathcal{C}_{\text {eq }}\left(\mathbf{k}, p_{i}, p_{j}\right)$ is the Fourier transformation of $\mathcal{C}_{\text {eq }}\left(\mathbf{x}_{i}-\mathbf{x}_{j}, p_{i}, p_{j}\right)$. To further proceed we choose the direction of $\mathbf{k}$ as the $x$-axis and integrate out $\omega$. It is natural to expect that $\mathcal{C}_{\text {eq }}\left(\mathbf{k}, p_{i}, p_{j}\right)$ possesses the spherical symmetry with respect to $\mathbf{p}_{i, j}$. As a result, we obtain

$$
\begin{array}{r}
\mathcal{C}_{\text {eq }}\left(\mathbf{k}, p_{1}, p_{2}\right)=\mathcal{C}_{\text {eq }}^{0}\left(\mathbf{k}, p_{1}, p_{2}\right)-\frac{4 \pi^{2} e^{2}}{k_{B} T} \frac{1}{|\mathbf{k}|^{2}} \frac{1}{p_{1 x}-p_{2 x}}\left\{p_{1 x} f_{\text {eq }}\left(p_{1}\right) \int d^{4} p_{3} u_{3}^{0} \mathcal{C}_{\text {eq }}\left(\mathbf{k}, p_{3}, p_{2}\right)\right. \\
\left.-p_{2 x} f_{\text {eq }}\left(p_{2}\right) \int d^{4} p_{3} u_{3}^{0} \mathcal{C}_{\text {eq }}\left(\mathbf{k}, p_{3}, p_{1}\right)\right\}
\end{array}
$$

Inserting Eq. (78) into it we find the solution to be

$$
C_{\text {eq }}\left(\mathbf{k}, p_{1}, p_{2}\right) \propto-\frac{f_{\text {eq }}\left(p_{1}\right) f_{\mathrm{eq}}\left(p_{2}\right)}{|\mathbf{k}|^{2}+\lambda_{\mathrm{D}}^{-2}} .
$$

Eq. (82) with $\lambda_{\mathrm{D}}$ given by Eq. (28) fully agrees with the result of Klimontovich who used a completely differ- 
(a)

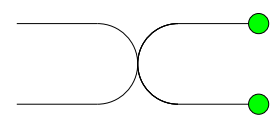

(b)

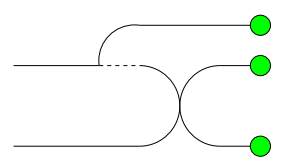

(c)

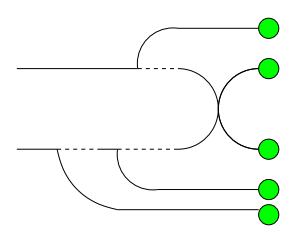

(d)

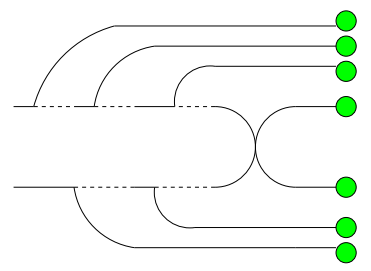

FIG. 3: Diagram of bare two-body correlation (a) and typical diagrams leading to short-ranged correlation (b)-(d). Solid circles stand for the Jüttner equilibrium.

ent (nonmanifestly covariant) approach [38]. They suggest that the equaltime two-body correlation function (in the phase space) displays an exponential decay with the correlation radius $\lambda_{\mathrm{D}}$, which is none but the nonrelativistic Debye length. The relativistic insensibility (rest mass) of $\lambda_{\mathrm{D}}$ was noticed long time ago [40]. Comparing Eq. (82) with Eq. (78) we find that despite of the appearance of the transverse electromagnetic interaction, which is screened in a dynamical manner [7, 41], the collective screening of the longitudinal electromagnetic interaction renders the equal-time correlation function short-ranged. Moreover, the static longitudinal permittivity is found to be

$$
\varepsilon^{\|}(\omega \rightarrow 0, \mathbf{k})-1=\frac{1}{\left(|\mathbf{k}| \lambda_{\mathrm{D}}\right)^{2}} .
$$

Thus, the ring diagrams suffice to describe the collective dielectric effects, justifying the introduced lower cutoff $\lambda_{\mathrm{D}}^{-1}$ in the relativistic Landau collision integral $\mathbb{K}_{1}[f]$. Of course, to heal the infrared divergence of $\mathbb{K}_{1}[f]$ accurately we need to sum up all the nonequilibrium ring diagrams as depicted in Fig. 1(b). This is technically far beyond the scope of the present paper and we leave for future studies.

\section{CONCLUSIONS}

The present work is motivated by very recent physical and mathematical progresses in 1-dimensional relativistic many-body systems [26, 42]. Although the attempts of reconciling the correlation dynamics and the special relativity were undertaken by the Brussel-Austin school long time ago, the relativistic correlation dynamics was formulated in a nonmanifestly covariant manner [12, 16]. Because of this drawback such a theory has been less advanced so far with regard to the practical applicability. The possibility of formulating a manifestly covariant correlation dynamics was first discussed by Israel and Kandrup [2, 3]. Unfortunately, there have been no progresses toward this direction. In this series of papers we substantially extend the analysis of the relativistic classical nonequilibrium statistical mechanics of full interacting many-body systems by Israel and Kandrup in the context of special relativity. Then, a manifestly covariant correlation dynamics results, which bridges macroscopic phenomena such as hydrodynamics and equilibrium and microscopic deterministic relativistic many-body dynamics. It is a statistical theory of ensembles of a bundle of world lines, and is suitable for studies of dynamics of distribution functions of full classical many-body systems. For a summary, we carry out the following program:

(i) For a classical interacting system composed of $N$ particles the well established action-at-a-distance formalism naturally introduces an $8 N$-dimensional $\Gamma$ phase space, for which we introduce a probability distribution function. From the conservation law a single-time Liouville equation follows, describing the manifestly covariant global evolution of the distribution function of the $\Gamma$ phase space.

(ii) For the manifestly covariant single-time Liouville equation we perform the correlation dynamics analysis. First, we introduce the correlation pattern representation, where the cluster expansion of reduced distribution functions is formulated in a manifestly covariant manner. Then, it is found that the evolution of the full $N$-body distribution function (of the $\Gamma$ phase space) may be reduced into that of the one-body distribution function (of the 8 -dimensional $\mu$ phase space) which is described by a closed nonlinear equation. However, the evolution of such a one-body distribution function may not be observable. Rather, the stationary solution of this closed nonlinear equation results in an exact general transport equation. The solution of the latter is physically observable and fully responsible for macroscopic hydrodynamics and, very importantly, determines the entire physical correlation functions. Such picture is encapsulated in Eqs. (17) and (19), which constitute a manifestly covariant version of the Bogoliubov functional assumption-the general principle underlying kinetic processes of manybody systems.

(iii) The collision integral of the general transport equation is expressed in terms of the density expansion. Therefore, for low-density systems only the leading term needs to be taken into account. For such obtained collision integral the perturbative theory with respect to the interaction is formulated. Then, under the relativistic impulse approximation we recover the Landau collision integral by keeping the leading order interaction expansion, while recover the Boltzmann collision integral by keeping the entire interaction expansion.

(iv) The analysis of the entropy production due to the Landau and the Boltzmann collision integral shows that a low-density system (for example, rarified plasmas) tend to be driven to the (local) Jüttner equilibrium. Replacing the physical one-body distribution function in the hierarchy (19) with the (global) Jüttner equilibrium, we justify the long standing many-body equilibrium conjecture of 
Hakim. In particular, we calculate explicitly the (static) two-body correlation function for plasmas and analyze the collective screening effects.

We remark that the emergence of a manifestly covariant formulation of the Bogoliubov functional assumption is by no means obvious. Indeed, in relativity the breakdown of the simultaneity as a covariant concept profoundly affects the mathematical structure of the correlation dynamics. Among the most important modifications is that the microscopic states, in general, are no longer the representation points in the $\Gamma$ phase space, rather, are a bundle of $N$ (segments of) particle world lines. (There do exist exceptions, for example, the case studied in Ref. [42].) Indeed, the introduction of the $\Gamma$ phase space is merely suggested by the form of the motion equations. In particular, that the force does not depend on the acceleration of the acted particle (Thus, the radiation reaction is ignored as electromagnetic interactions concerned.) is crucial to the present choice of the $\Gamma$ phase space. As a result, the building block operator namely the two-body interacting Liouvillian and the distribution function are determined not only by the positions and the momenta of two participating particles, but also by their world lines (more precisely, the phase trajectories) passing through the given positions and momenta. The very origin of the Bogoliubov functional assumption namely Eqs. (17) and (19) is that the deterministic many-body dynamics lose the memory of the given (segments of) particle world lines at large global proper times.

In fact, it is the nonmanifestly covariant version of the Bogoliubov functional assumption, formulated in a rather straightforward manner, that is commonly adopted in the literature of plasmas $18,43,44]$. However, the proof has been lacking. The manifestly covariant Bogoliubov functional assumption namely Eqs. (17) and (19) justifies such an formulation. Indeed, it was first noticed by Dirac, Fock, and Podolsky [45] and stressed by Israel [3] that it is legitimate to identify the coordinate times of particles, i.e., to set $t_{1}=t_{2}=\cdots=t_{N} \equiv t$ for the manifestly covariant equation $\hat{\mathfrak{L}} \mathcal{N}=0$. In doing so we obtain Eqs. (17) and (18) with all the coordinate times set to be the same. Next, as (implicitly) done in various nonmanifestly covariant theories we again apply the relativistic impulse approximation. The key point is that such an approximation does not destroy the (manifest) covariance of Lorentz force. Then, the collision integral (33) remains unaffected except that there $t_{1}$ and $t_{2}$ have to be identified. For this collision integral let us keep only the leading order interaction expansion. Immediately, we arrive at a relativistic Landau collision integral which is a nonmanifestly covariant version of $\mathbb{K}_{1}[f]$. Thus, we explain the insightful observation made in Ref. [2], where it was pointed out that to derive relativistic transport equations by using a nonmanifestly covariant approach may not be a problem as electromagnetic interactions concerned.

We stress that upon passing from the motion equations (11) and (2) to the single-time Louville equation (5) some information of the underlying many-body dynamics is lost. Thus, they are not equivalent. The manifestly covariant correlation dynamics stems from the latter. Surprisingly, for rarefied plasmas the present correlation dynamics recovers the Landau and the Boltzmann equation which are fully responsible for various macroscopic phenomena such as hydrodynamics and the (local) equilibrium. Thus, we are led to the following conjecture:

For systems with arbitrary macroscopic particle number density the manifestly covariant single-time Louville equation (5) completely determines macroscopic relativistic hydrodynamics.

Although the relativistic Landau equation has been justified before especially by the manifestly covariant nonequilibrium statistical mechanics developed in Refs. 2, [3], compared to the earlier theories the manifestly covariant correlation dynamics presented here has the additional advantage in going beyond the weak coupling approximation: First, it clearly shows that the relativistic Landau equation is the leading order interaction expansion of the relativistic Boltzmann equation. It is the two-body scattering approximation that is used in deriving the latter equation, and the collision integral (with the accuracy of the leading order density expansion) is expressed in terms of the interaction expansion. The present theory allows one to sum up this expansion and, as a result, the scattering cross section is beyond the weak coupling level. Second, the general collision integral (18) and the hierarchy of correlation functions (19) further allow one to systematically go beyond the two-body scattering approximation. This is exemplified in the calculations of the static two-body equilibrium correlation. It is promising in understanding the infrared divergence of the relativistic Landau collision integral where collective dynamic screening phenomena play crucial roles, and in generalizing the Balescu-Lenard equation [20] so as to suit relativity principles. (To our best knowledge, so far only the nonmanifestly covariant Balescu-Lenard equation has been obtained [18, 38, 46].) Finally, the present theory allows one to explore the anomalous transport phenomenon arising from the long-time tail correlation effects [47]. These issues seem to be far beyond the reach of earlier theories such as Refs. [2, 3].

Closing this paper we remark that although this series of papers focuses on the classical correlation dynamics, it may have important implications on the relativistic quantum nonequilibrium statistical mechanics. Indeed, to formulate the latter at the level of full many-body quantum dynamics has been of long term interests and remained controversial so far [48]. Besides of the manifest/nonmanifest covariance issue another severe difficulty arises as the quantization of relativistic many-body classical dynamics concerned. In order not to lose the manifest covariance Schieve recently started from the socalled covariant Hamiltonian dynamics and formulated a theory of relativistic quantum statistical mechanics [48]. 
It is important to notice that the quantum Boltzmann equation derived there is with regard to "events" in the spacetime rather than particles. For this reason neither can this equation be justified by the microscopic approach [14] basing on the generalization of traditional nonequilibrium Green's function theories [49, 50] or by refined relativistic nonequilibrium quantum field theories [15, 25], nor the classical limit of this equation be justified by other classical microscopic theories [2, 3]. In contrast, the quantum transport equations obtained by complete quantum treatment, in the classical limit, agree with the transport equations shown in the present paper. This justifies the legitimacy of basing classical relativistic statistical mechanics on the action-at-a-distance formalism. Many natural problems thereby arise: May it be possible to be extended to the quantum case? What is the quantum counterpart of the proper time-parametrized evolution? In particular, over a field theory the advantage of proceeding along this line to formulate a quantum statistical theory is to allow for nonperturbative treatment and to encompass some global properties, as admitted by many authors (for example, see Ref. [15]). Unfortunately, to solve these problems is by no means an easy task because of the lack of Hamiltonian of the action-at-adistance formalism. A possible prescription is to enlarge the configuration space to accommodate the field degrees of freedom. Treating the particles and the fields on the same footing one may proceed to construct a many-body Green function by adopting the prescription of Feynman [51, 52], namely to express it in terms of the functional integral over all the paths of both particles and fields parametrized by separate proper times. Furthermore, it is expected that such a many-body Green function satisfies a Stückelberg-type equation [53] suitable for the use of the Wigner function technique. As such the entire theoretical scope of nonrelativistic quantum correlation dynamics [34] might become applicable. We leave the detailed analysis for future studies.

\section{Acknowledgments}

I am deeply grateful to Q. K. Lu for numerous fruitful discussions at the early stage of this work, and especially to S. L. Tian for invaluable help. I also would like to thank M. Courbage, J. R. Dorfman and M. Garst for useful conversations, and especially to C. Kiefer for his interests and encouragements. This work is supported by Transregio SFB 12 of the Deutsche Forschungsgemeinschaft and was partly done in Institute of Henri Poincare.

\section{APPENDIX A: RELATIVISTIC IMPULSE APPROXIMATION FOR ELECTROMAGNETIC INTERACTIONS}

In this appendix we wish to study the relativistic impulse approximation to two-body interacting Liouvillian for classical electromagnetic interactions.

\section{Exact two-body interacting Liouvillian}

We notice that, in general, the interacting force $F_{i j}^{\mu}$ has the form as follows [23, 54]:

$$
\begin{gathered}
F_{i j}^{\mu}\left(x_{i}, p_{i}\right)=\left.\int_{-\infty}^{+\infty} d \tau_{j} s\left(\rho_{i j}\right) \mathcal{F}^{\mu \nu}\right|_{x_{j}=x_{j}\left(\tau_{j}\right)} p_{\nu i}, \\
\rho_{i j}=\left(x_{\mu i}-x_{\mu j}\right)\left(x_{i}^{\mu}-x_{j}^{\mu}\right)
\end{gathered}
$$

where $\mathcal{F}^{\mu \nu}$ is an antisymmetric tensor, and the role of function $g\left(\rho_{i j}\right)$ is to invariantly connect $x_{i}$ with one (or several) points at the world line $x_{j}\left(\tau_{j}\right)$. Let us exemplify Eq. (A1) and derive the exact form of two-body interacting Liouvillian in classical electrodynamics. For this purpose we employ the Wheeler-Feynman formalism 32. and start from the Fokker action which crucially allows us to eliminate the self-action:

$$
S=-m \sum_{i} \int d s_{i}\left(\bar{u}_{\mu i} \bar{u}_{i}^{\mu}\right)^{1 / 2}-e^{2} \sum_{i<j} \iint d s_{i} d s_{j} \bar{u}_{\mu i} \bar{u}_{j}^{\mu} \delta\left(\rho_{i j}\right)
$$

with $\bar{u}^{\mu}(s) \equiv d x^{\mu}(s) / d s$.

Then, we apply the variation principle to this action. Demanding

$$
\delta S=m \sum_{i} \int d s_{i} \frac{d}{d s_{i}} \frac{\bar{u}_{i}^{\mu}}{\sqrt{\bar{u}_{\nu i} \bar{u}_{i}^{\nu}}} \delta x_{\mu i}+e^{2} \sum_{i \neq j} \iint d s_{i} d s_{j}\left\{\partial_{i}^{\nu}\left[\bar{u}_{j}^{\mu} \delta\left(\rho_{i j}\right)\right]-\partial_{i}^{\mu}\left[\bar{u}_{j}^{\nu} \delta\left(\rho_{i j}\right)\right]\right\} \bar{u}_{\nu i} \delta x_{\mu i} \equiv 0
$$

and defining $d \tau_{i}=\sqrt{\bar{u}_{\nu i} \bar{u}_{i}^{\nu}} d s_{i}$ and $u_{i}^{\mu} \equiv \bar{u}_{i}^{\mu} / \sqrt{\bar{u}_{\nu i} \bar{u}_{i}^{\nu}}$, we obtain

$$
\begin{aligned}
\frac{d p_{i}^{\mu}}{d \tau_{i}} & =e \sum_{j \neq i} F_{i j}^{\mu \nu}\left(x_{i}\right) u_{\nu i} \\
F_{i j}^{\mu \nu}\left(x_{i}\right) & =\left.\left[\partial^{\mu} A_{j}^{\nu}(x)-\partial^{\nu} A_{j}^{\mu}(x)\right]\right|_{x=x_{i}},
\end{aligned}
$$

where $F_{i j}^{\mu \nu}$ is the electromagnetic tensor adjunct to particle $j$, and all the field point is set to be the position 
of particle $i$ at $\tau_{i}$, i.e., $x=x_{i}\left(\tau_{i}\right)$. The vector potential, adjunct to particle $j$, is given by

$$
A_{j}^{\mu}(x)=e \int \delta\left(\left(x-x_{j}\right)^{2}\right) u_{j}^{\mu} d \tau_{j} .
$$

Notice that here both $x_{j}$ and $u_{j}$ depend on the proper time $\tau_{j}$. Eqs. (A44) and (A5) describe the classical electrodynamics of electronic systems in terms of direct interparticle action. Notice that the vector potential given by Eq. A5 satisfies Lorentz gauge:

$$
\partial_{\mu} A_{j}^{\mu}(x)=0
$$

Inserting Eq. A5 into Eq. (A4) gives Eq. (A1) with

$$
\begin{gathered}
\mathcal{F}^{\mu \nu}=-\frac{e^{2}}{m^{2}}\left[\left(x_{i}^{\mu}-x_{j}^{\mu}\right) p_{j}^{\nu}-p_{j}^{\mu}\left(x_{i}^{\nu}-x_{j}^{\nu}\right)\right], \\
s\left(\rho_{i j}\right)=\delta^{\prime}\left(\rho_{i j}\right) .
\end{gathered}
$$

Here $\delta^{\prime}(x) \equiv d \delta(x) / d x$.

With the help of Eqs. (A4 and (A5) the exact twobody interacting Liouvillian is written as

$$
\begin{aligned}
\lambda \hat{\mathcal{L}}_{i j}^{\prime}\left(x_{i}, p_{i} ; x_{j}, p_{j}\right)=-e^{2} \int d \tau & \left\{u_{\nu i}\left[u_{j}^{\nu}(\tau) \partial_{i}^{\mu}-u_{j}^{\mu}(\tau) \partial_{i}^{\nu}\right] \delta\left(\left(x_{i}-x_{j}(\tau)\right)^{2}\right) \frac{\partial}{\partial p_{i}^{\mu}}\right. \\
& \left.+u_{\nu j}\left[u_{i}^{\nu}(\tau) \partial_{j}^{\mu}-u_{i}^{\mu}(\tau) \partial_{j}^{\nu}\right] \delta\left(\left(x_{j}-x_{i}(\tau)\right)^{2}\right) \frac{\partial}{\partial p_{j}^{\mu}}\right\} .
\end{aligned}
$$

\section{Two-body interacting Liouvillian: relativistic impulse approximation}

Now we proceed to find the relativistic impulse approximation to the two-body interacting Liouvillian. For this purpose we use the Dirac identity: $\partial_{\mu} \partial^{\mu} \delta\left(x_{\mu} x^{\mu}\right)=4 \pi \delta^{(4)}(x)$ to obtain

$$
\delta\left(x_{\mu} x^{\mu}\right)=-4 \pi \int \frac{d^{4} k}{(2 \pi)^{4}} \frac{e^{i k \cdot x}}{k^{2}} .
$$

Substituting it into Eq. (A9) and integrating out $\tau$, with Eq. (34) taken into account we obtain

$$
F_{i j}^{\mu}\left(x_{i}, p_{i}\right)=-\frac{i 8 \pi^{2} e^{2}}{m} \int \frac{d^{4} k}{(2 \pi)^{4}} \frac{e^{i k \cdot\left(x_{i}-x_{j}\right)}}{k^{2}} \delta\left(k \cdot p_{j}\right)\left[k^{\mu}\left(p_{i} \cdot p_{j}\right)-p_{j}^{\mu}\left(k \cdot p_{i}\right)\right]
$$

and likewise for $F_{i i}^{\mu}\left(x_{j}, p_{j}\right)$. Eventually we find the twobody interacting Liouvillian, under the relativistic impulse approximation to be Eq. (36). (Notice that there we need to make the replacement: $1 \rightarrow i, 2 \rightarrow j$ for the subscript.) It is important to notice that the mass-shell constraint is preserved by the forces:

$$
F_{i j} \cdot p_{i}=F_{j i} \cdot p_{j}=0
$$

\section{Two-body scattering in the CM frame}

Suppose that the coordinate times of particle $i$ and $j$ are identified, i.e., $t_{i}=t_{j} \equiv t$. Then, in order for Eq. (54) to accommodate a solution in the CM frame we need to establish the following $\left(\gamma_{i, j}=\sqrt{m^{2}+\left|\mathbf{p}_{i, j}\right|^{2}} / m\right)$

Lemma. For the relativistic two-body dynamics de- scribed by the following equations:

$$
\begin{aligned}
\gamma_{i} \frac{d \mathbf{x}_{i}}{d t} & =\frac{\mathbf{p}_{i}}{m}, & \gamma_{i} \frac{d \mathbf{p}_{i}}{d t} & =\mathbf{F}_{i j} \\
\gamma_{j} \frac{d \mathbf{x}_{j}}{d t} & =\frac{\mathbf{p}_{j}}{m}, & \gamma_{j} \frac{d \mathbf{p}_{j}}{d t} & =\mathbf{F}_{j i}
\end{aligned}
$$

with the force given by Eq. (A11), if at $t=0$ exists $\mathbf{p}_{i}(0)+\mathbf{p}_{j}(0)=0$, then it holds for all $t>0$, i.e., $\mathbf{p}_{i}(t)+\mathbf{p}_{j}(t)=0$.

Proof. This is quite obvious. First of all, enforcing $t_{i}=t_{j} \equiv t$ we find, from Eq. (A11), that the force arising from interactions depends on $\mathbf{x}_{i}-\mathbf{x}_{j}$ and $\mathbf{p}_{i, j}$. Then, Eqs. (A13) and (A14) uniquely determine the phase trajectories: $\mathbf{x}_{i, j}(t)$ provided that the initial condition: $\mathbf{x}_{i, j}(0), \mathbf{p}_{i, j}(0)$ is set.

On the other hand, consider the trajectories satisfying $\mathbf{x}_{i}(t)-\mathbf{x}_{0}=-\left(\mathbf{x}_{j}(t)-\mathbf{x}_{0}\right) \equiv \mathbf{x}(t)$, where $\mathbf{x}_{0} \equiv\left(\mathbf{x}_{i}(0)+\right.$ $\left.\mathbf{x}_{j}(0)\right) / 2$. We find that they solve Eqs. (A13) and (A14) 
by noticing

$$
\begin{aligned}
\mathbf{F}_{i j}=\frac{8 \pi^{2} e^{2}}{m^{2}} & \int_{|\mathbf{k}| \gtrsim_{\mathrm{D}}^{-1}} \frac{d^{3} \mathbf{k}}{(2 \pi)^{4}} \frac{\sin (2 \mathbf{k} \cdot \mathbf{x})}{(\mathbf{k} \cdot \mathbf{p})^{2}-|\mathbf{k}|^{2} E^{2}} \\
& \times E\left[\mathbf{k}\left(E^{2}+|\mathbf{p}|^{2}\right)-2(\mathbf{k} \cdot \mathbf{p}) \mathbf{p}\right],
\end{aligned}
$$

where $\mathbf{p}(t) \equiv \mathbf{p}_{i}(t)=-\mathbf{p}_{j}(t)$ satisfying $\mathbf{p}=\gamma m \dot{\mathbf{x}}$ $\left(\gamma=\sqrt{m^{2}+|\mathbf{p}|^{2}} / m\right)$, and $E \equiv \sqrt{m^{2}+|\mathbf{p}|^{2}}$. Thus, Eqs. A13 and A14 do admit the solution satisfying $\mathbf{p}_{i}(t)+\mathbf{p}_{j}(t)=0$ irrespective of $\mathbf{x}_{i, j}(0)$. With the uniqueness taken into account the lemma follows. Q.E.D.

Remark. The lemma shows that under the relativistic impulse approximation the two-body scattering may be described in the usual CM frame where $\mathbf{p}_{i}(t)+\mathbf{p}_{j}(t)=0$ and $\mathbf{x}_{i}(t)+\mathbf{x}_{j}(t)=0$. Importantly, in this frame the two-body dynamics may be reduced into the dynamics of single particle subject to the external force given by Eq. (A15.

\section{APPENDIX B: RELATIVISTIC LANDAU COLLISION INTEGRAL}

We now calculate Eq. (40) under the relativistic impulse approximation. For this purpose we insert the two-body interacting Liouvillian namely Eq. (36) into it. Notice that $\lambda \hat{\mathcal{L}}_{i j}^{\prime}\left(x_{i}, p_{i} ; x_{j}, p_{j}\right)$ depends on $x_{i, j}$ through $x_{i}-x_{j}$. Then,

$$
\begin{aligned}
\mathbb{K}_{1}[f]= & -\left(\frac{8 \pi^{2} e^{2}}{m}\right)^{2} \int_{0}^{\infty} d s \int_{\Sigma_{2} \otimes U_{2}^{4}} d \Sigma_{\mu 2} d^{4} p_{2} u_{2}^{\mu} \iint \frac{d^{4} k}{(2 \pi)^{4}} \frac{d^{4} k^{\prime}}{(2 \pi)^{4}} \\
& \times e^{i k \cdot\left(x_{1}-x_{2}\right)} \hat{\mathcal{G}}_{12}(k) e^{i k^{\prime} \cdot\left\{x_{1}-x_{2}-s\left(u_{1}-u_{2}\right)\right\}} \hat{\mathcal{G}}_{12}\left(k^{\prime}\right) f\left(x_{1}, p_{2}\right) f\left(x_{1}, p_{1}\right) \\
= & -\pi\left(\frac{8 \pi^{2} e^{2}}{m}\right)^{2} \int_{\Sigma_{2} \otimes U_{2}^{4}} d \Sigma_{\mu 2} d^{4} p_{2} p_{2}^{\mu} \iint \frac{d^{4} k}{(2 \pi)^{4}} \frac{d^{4} k^{\prime}}{(2 \pi)^{4}} \\
& \times e^{i k \cdot\left(x_{1}-x_{2}\right)} \hat{\mathcal{G}}_{12}(k) e^{i k^{\prime} \cdot\left(x_{1}-x_{2}\right)} \delta\left(k^{\prime} \cdot p_{1}-k^{\prime} \cdot p_{2}\right) \hat{\mathcal{G}}_{12}\left(k^{\prime}\right) f\left(x_{1}, p_{2}\right) f\left(x_{1}, p_{1}\right),
\end{aligned}
$$

where in the derivation of the second equality we have integrated out $s$. Inserting the expression of $\hat{\mathcal{G}}_{12}\left(k^{\prime}\right)[$ see Eq. (36)] into it we obtain

$$
\begin{aligned}
\mathbb{K}_{1}[f]= & -\pi\left(\frac{8 \pi^{2} e^{2}}{m}\right)^{2} \int_{\Sigma_{2} \otimes U_{2}^{4}} d \Sigma_{\mu 2} d^{4} p_{2} p_{2}^{\mu} \iint \frac{d^{4} k}{(2 \pi)^{4}} \frac{d^{4} k^{\prime}}{(2 \pi)^{4}} \\
& \times e^{i k \cdot\left(x_{1}-x_{2}\right)} \hat{\mathcal{G}}_{12}(k) e^{i k^{\prime} \cdot\left(x_{1}-x_{2}\right)} \delta\left(k^{\prime} \cdot p_{1}\right) \delta\left(k^{\prime} \cdot p_{2}\right)\left(p_{1} \cdot p_{2}\right) \frac{k^{\prime \mu}}{k^{\prime 2}}\left(\frac{\partial}{\partial p_{1}^{\mu}}-\frac{\partial}{\partial p_{2}^{\mu}}\right) f\left(x_{1}, p_{2}\right) f\left(x_{1}, p_{1}\right) .
\end{aligned}
$$

Let us carry out the spatial integral first. For this purpose we enjoy the manifest covariance and choose $\Sigma_{2}$ to be the usual 3-dimensional Euclidean space. As a result, we obtain

$$
\int_{\Sigma_{2}} d \Sigma_{\mu 2} e^{i\left(k+k^{\prime}\right) \cdot\left(x_{1}-x_{2}\right)}=(2 \pi)^{3} \delta^{(3)}\left(\mathbf{k}+\mathbf{k}^{\prime}\right) e^{i\left(\omega+\omega^{\prime}\right)\left(t_{1}-t_{2}\right)} .
$$

where we keep in mind that the wave vector $k^{\mu} \equiv(\omega, \mathbf{k})$. Then,

$$
\begin{aligned}
& \int_{\Sigma_{2}} d \Sigma_{\mu 2} p_{1}^{\mu} e^{i\left(k+k^{\prime}\right) \cdot\left(x_{1}-x_{2}\right)} \delta\left(k \cdot p_{1}\right) \delta\left(k^{\prime} \cdot p_{1}\right) \delta\left(k^{\prime} \cdot p_{2}\right) \\
= & \int_{\Sigma_{2}} d \Sigma_{\mu 2} p_{2}^{\mu} e^{i\left(k+k^{\prime}\right) \cdot\left(x_{1}-x_{2}\right)} \delta\left(k \cdot p_{2}\right) \delta\left(k^{\prime} \cdot p_{1}\right) \delta\left(k^{\prime} \cdot p_{2}\right)=(2 \pi)^{3} \delta^{(4)}\left(k+k^{\prime}\right) \delta\left(k \cdot p_{1}\right) \delta\left(k \cdot p_{2}\right) \\
& \int_{\Sigma_{2}} d \Sigma_{\mu 2} k^{\mu} e^{i\left(k+k^{\prime}\right) \cdot\left(x_{1}-x_{2}\right)} \delta\left(k \cdot p_{1}\right) \delta\left(k^{\prime} \cdot p_{1}\right) \delta\left(k^{\prime} \cdot p_{2}\right)=0
\end{aligned}
$$

Let us substitute the expression of $\hat{\mathcal{G}}_{12}(k)$ into Eq. (B2) and take Eqs. (B4) and (B5) into account. Noticing

$$
\left[\delta\left(k \cdot p_{2}\right) k^{\mu}\left(p_{1} \cdot p_{2}\right), \partial / \partial p_{1}^{\mu}\right]=\left[\delta\left(k \cdot p_{1}\right) k^{\mu}\left(p_{1} \cdot p_{2}\right), \partial / \partial p_{2}^{\mu}\right]=0
$$

we arrive at Eq. (41). 


\section{APPENDIX C: DIVERGENCE OF TENSOR $\epsilon^{\mu \nu}$}

Observing Eq. (42) it is easy to see that $\epsilon^{\mu \nu}$ possesses the following structure

$$
\epsilon^{\mu \nu}=c_{1} g^{\mu \nu}+c_{2}\left(u_{1}^{\mu} u_{1}^{\nu}+u_{2}^{\mu} u_{2}^{\nu}\right)+c_{3}\left(u_{1}^{\mu} u_{2}^{\nu}+u_{2}^{\mu} u_{1}^{\nu}\right)
$$

enforced by the Lorentz invariance, with $c_{1,2,3}$ the coefficients which are invariant under the Lorentz transformation. Then, from the identity: $p_{1 \mu} \epsilon^{\mu \nu}=p_{2 \mu} \epsilon^{\mu \nu}=0$ we find $c_{1}=\left[\left(u_{1} \cdot u_{2}\right)^{2}-1\right] c_{2}, c_{3}=-\left(u_{1} \cdot u_{2}\right) c_{2}$. Substituting these two relations into Eq. (C1) gives

$$
\epsilon^{\mu \nu}=c_{2}\left\{\left[\left(u_{1} \cdot u_{2}\right)^{2}-1\right] g^{\mu \nu}+\left(u_{1}^{\mu} u_{1}^{\nu}+u_{2}^{\mu} u_{2}^{\nu}\right)-\left(u_{1} \cdot u_{2}\right)\left(u_{1}^{\mu} u_{2}^{\nu}+u_{2}^{\mu} u_{1}^{\nu}\right)\right\}
$$

where $c_{2}$ is determined by

$$
c_{2}=\frac{1}{2}\left[\left(u_{1} \cdot u_{2}\right)^{2}-1\right]^{-1} g_{\mu \nu} \epsilon^{\mu \nu} .
$$

We now come to calculate $c_{2}$. For this purpose we insert Eq. (42) into Eq. (C3) arriving at

$$
c_{2}=e^{4} \frac{\left(u_{1} \cdot u_{2}\right)^{2}}{\left(u_{1} \cdot u_{2}\right)^{2}-1} \int d^{4} k \delta\left(k \cdot u_{1}\right) \delta\left(k \cdot u_{2}\right) \frac{1}{k \cdot k} .
$$

To proceed further we enjoy the Lorentz invariance and choose the direction of $\mathbf{u}_{1}$ as the $x$-axis of the 3 -dimensional Euclidean space. With $\omega$ integrated out we find

$$
\begin{aligned}
c_{2} & =e^{4} \frac{\left(u_{1} \cdot u_{2}\right)^{2}}{\gamma_{1} \gamma_{2}\left[\left(u_{1} \cdot u_{2}\right)^{2}-1\right]} \int d^{3} \mathbf{k} \frac{\delta\left(k_{x}\left(v_{1}-v_{2 x}\right)-k_{y} v_{2 y}-k_{z} v_{2 z}\right)}{\left(k_{x} v_{1}\right)^{2}-\mathbf{k}^{2}} \\
& =-e^{4} \frac{\left(p_{1} \cdot p_{2}\right)^{2}}{\gamma_{1} \gamma_{2}\left[\left(u_{1} \cdot u_{2}\right)^{2}-1\right]} \frac{1}{\left|v_{1}-v_{2 x}\right|} \int d^{2} k_{\perp} \frac{1}{\left[\frac{\mathbf{k}_{\perp} \cdot \mathbf{v}_{2 \perp}}{\gamma_{1}\left(v_{1}-v_{2 x}\right)}\right]^{2}+\mathbf{k}_{\perp}^{2}},
\end{aligned}
$$

where in the last line we use the notation: $\mathbf{k}_{\perp}=\left(k_{y}, k_{z}\right), \mathbf{v}_{2 \perp}=\left(v_{2 y}, v_{2 z}\right)$.

The integral in the last line may be easily performed by passing to the polar coordinate system, i.e., $\left(k_{\perp}, \theta\right)$ with the axis chosen to be the direction of $\mathbf{v}_{2 \perp}$. As such the integral is factorized into the integral over $\theta$ which is finite [55], and the integral over $k_{\perp}$ which suffers from both the ultraviolet and the infrared divergence. More precisely,

$$
\begin{aligned}
\int d^{2} k_{\perp} \frac{1}{\left[\frac{\mathbf{k}_{\perp} \cdot \mathbf{v}_{2 \perp}}{\gamma_{1}\left(v_{1}-v_{2 x}\right)}\right]^{2}+\mathbf{k}_{\perp}^{2}} & =\int \frac{d k_{\perp}}{k_{\perp}} \int_{0}^{2 \pi} d \theta \frac{1}{\left(1+\frac{\mathbf{v}_{2 \perp}^{2}}{\gamma_{1}^{2}\left(v_{1}-v_{2 x}\right)^{2}} \cos ^{2} \theta\right)} \\
& =\frac{2 \pi}{\sqrt{1+\frac{\mathbf{v}_{2 \perp}^{2}}{\gamma_{1}^{2}\left(v_{1}-v_{2 x}\right)^{2}}}} \int \frac{d k_{\perp}}{k_{\perp}} .
\end{aligned}
$$

We see that, indeed, a logarithmic divergence results. Substituting it into Eq. (C5) gives

$$
\begin{aligned}
c_{2} & =-2 \pi e^{4} \int \frac{d k_{\perp}}{k_{\perp}} \frac{\left(u_{1} \cdot u_{2}\right)^{2}}{\gamma_{1} \gamma_{2}\left[\left(u_{1} \cdot u_{2}\right)^{2}-1\right]} \frac{1}{\sqrt{\left(v_{1}-v_{2 x}\right)^{2}+\mathbf{v}_{2 \perp}^{2}\left(1-v_{1}^{2}\right)}} \\
& =-2 \pi e^{4} \int \frac{d k_{\perp}}{k_{\perp}} \frac{\left(u_{1} \cdot u_{2}\right)^{2}}{\gamma_{1} \gamma_{2}\left[\left(u_{1} \cdot u_{2}\right)^{2}-1\right]} \frac{1}{\sqrt{-\left(1-v_{1}^{2}\right)\left(1-v_{2}^{2}\right)+\left(1-v_{1} v_{2 x}\right)^{2}}} .
\end{aligned}
$$

Taking into account the identity: $u_{1} \cdot u_{2}=\gamma_{1} \gamma_{2}\left(1-v_{1} v_{2 x}\right) \quad$ Inserting it into Eq. (C2) we arrive at Eq. (43). eventually we find

$$
c_{2}=-2 \pi e^{4} \int \frac{d k_{\perp}}{k_{\perp}} \frac{\left(u_{1} \cdot u_{2}\right)^{2}}{\left[\left(u_{1} \cdot u_{2}\right)^{2}-1\right]^{3 / 2}} .
$$

[1] G. Chacon-Acosta and G. M. Kremer, Phys. Rev. E 76, 021201 (2007); J. Ramos-Caro and G. A. Gonzalez, Class. 
[2] W. Israel and H. E. Kandrup, Ann. Phys. (N.Y.) 152, 30 (1984).

[3] H. E. Kandrup, Ann. Phys. (N.Y.) 153, 44 (1984).

[4] H. E. Kandrup, Ann. Phys. (N.Y.) 169, 352 (1986).

[5] For examples, see: A. Bret, L. Gremillet, D. Benisti, and E. Lefebvre, Phys. Rev. Lett. 100, 205008 (2008); T. Yokota, Y. Nagao, T. Johzaki, and K. Mima, Phys. Plasmas, 13, 022702 (2006).

[6] U. Heinz, Phys. Rev. Lett. 51, 351 (1983).

[7] D. F. Litim and C. Manuel, Phys. Rep. 364, 451 (2002); J.-P. Blaizot and E. Iancu, ibid. 359, 355 (2002).

[8] M. Müller and S. Sachdev, Phys. Rev. B 78, 115419 (2008).

[9] R. Hakim, J. Math. Phys. 8, 1315 (1967); 8, 1379 (1967).

[10] C. Cercigani and G. M. Kremer, The relativistic Boltzmann equation: Theory and applications (Birkhäuser, Basel, 2002).

[11] S. R. de Groot, W. A. van Leeuwen, and Ch. G. van Wheert, Relativistic kinetic theory (North-Holland, Amsterdam, 1980).

[12] I. Prigogine, in Statistical mechanics of equilibrium and nonequilibrium, J. Meixner ed., (North-Holland, Amsterdam, 1965).

[13] Yu. L. Klimontovich, Zh. Eksp. Teor. Fiz., 37, 735 (1959); 38, 1212 (1960) [Sov. Phys. JETP 10, 524 (1960); 11, 876 (1960)].

[14] B. Bezzerides and D. F. DuBois, Ann. Phys. (N.Y.) 70, 10 (1972).

[15] E. Calzetta and B. L. Hu, Phys. Rev. D 37, 2878 (1988).

[16] R. Balescu, in 1964 Cargese Summer School (Gordon and Breach, New York, 1965); R. Balescu and T. Kotera, Physica 33, 558 (1967).

[17] L. P. Horwitz, S. Shashoua, and W. C. Schieve, Physica 161A, 300 (1989).

[18] Q. Lu, Chin. Phys. Lett. 10, 69 (1993); Sci. Sinica A 37, 1241 (1994); Chin. Sci. Bull. 40, 1314 (1995).

[19] U. Ben-Ya'acov, Physica 222 A, 307 (1995).

[20] R. Balescu, Phys. Fluids. 3, 52 (1960); A. Lenard, Ann. Phys. (N.Y.) 3, 390 (1960).

[21] I. Prigogine and G. Severne, Physica 32, 1376 (1966).

[22] Yu. Kukharenko, A. Vityazev, and A. Bashkirov, Phys. Lett. A 195, 27 (1994).

[23] C. Tian, the preceeding paper.

[24] In order not to make any confusion we here distinguish such a general kinetic equation from the kinetic equation of Boltzmann and the Boltzmann-like equation in both nonrelativistic and relativistic physics. The former, derived from the many-body Liouville equation, is exact, closed, and highly nonlinear. It is satisfied by the reduced one-body distribution function. The kinetic equation of Boltzmann is a special case of this general kinetic equation under appropriate approximations [33, 34]. Notice that in many condensed matter and high-energy literatures (for examples, Refs. [7, 25, 50]) the "Boltzmann" equation is in fact "Boltzmann-like" which, typically, is the kinetic (transport) equation of Vlasov, FokkerPlanck, Landau, or of Boltzmann but with the scattering cross section calculated at the level of the weak coupling (Born) approximation.

[25] K. C. Chou, Z. B. Su, B. L. Hao, and L. Yu, Phys. Rep. 118, 1 (1985).

[26] D. Cubero, J. Casado-Pascual, J. Dunkel, P. Talker, and P. Hänggi, Phys. Rev. Lett. 99, 170601 (2007).
[27] F. Debbasch, Physica 387 A, 2443 (2008) and references therein.

[28] J. Dunkel and P. Hänggi, Phys. Rep. 471, 1 (2009) and references therein.

[29] R. M. Dudley, Ark. Mat. Astron. Fys. 6, 241 (1965); 6, 575 (1967).

[30] R. Hakim, J. Math. Phys. 9, 1805 (1968).

[31] J. Dunkel, P. Hänggi, and S. Weber Phys. Rev. E 79, 010101 (2009).

[32] A. D. Fokker, Z. Physik 58, 386 (1929); J. A. Wheeler and R. P. Feynman, Rev. Mod. Phys. 21, 425 (1949); see also D. J. Louis-Martinez, Phys. Lett. B 632, 733 (2006) for a recent review.

[33] I. Prigogine, Non-equilibrium statistical mechanics (Wiley, New York, 1963).

[34] R. Balescu, Equilibrium and nonequilibrium statistical mechanics (Wiley, New York, 1975).

[35] N. N. Bogoliubov, in Studies in statistical mechanics I, J. de Boer and G. E. Uhlenbeck eds., (North-Holland, Amsterdam, 1962).

[36] S. T. Belyaev and G. Budker, Dokl. Akad. Nauk SSSR 107, 807 (1956) [Sov. Phys.-Doklady 1, 218 (1957)].

[37] P. Clavin, C. R. Acad. Sc. (Paris) 274A, 1022 (1972); 274A, 1085 (1972).

[38] Yu. L. Klimontovich, The statistical theory of nonequilibrium process in a plasma (MIT, Cambridge, 1967).

[39] L. D. Landau, Zh. Eksp. Teor. Fiz. 7, 203 (1937); In Collected papers of L. D. Landau (Pergamon Press, Oxford, 1965).

[40] L. D. Landau and E. M. Lifshitz, Physical kinetics 1st ed. (Pergamon Press, Oxford, 1981).

[41] A. M. Tsvelik, Quantum field theory in condensed matter physics (Cambridge, UK, 1995).

[42] S. B. Kirpichev and P. A. Polyakov, J. Math. Sci. 141, 1051 (2007).

[43] N. D. Naumov, Izv. Vyssh. Uchebn. Zaved. Fiz. 3, 78 (1981).

[44] C. Tian, C. Zhang, and Q. Lu, Comm. Theor. Phys. 35, 605 (2001).

[45] P. A. M. Dirac, V. A. Fock, and B. Podolsky, Phys. Z. Sowjetunion 2, 468 (1932).

[46] Yu. A. Markov and M. A. Markova, Teor. Mat. Fiz. 103, 123 (1995) [Theor. Math. Phys. 103, 444 (1995)].

[47] J. R. Dorfman and E. G. D. Cohen, Phys. Rev. Lett. 25, 1257 (1970); M. H. Ernst, E. H. Hauge, and J. M. J. van Leeuwen, ibid. 25, 1254 (1970).

[48] W. C. Schieve, Found. Phys. 35, 1359 (2005).

[49] J. Schwinger, J. Math. Phys. 2, 407 (1961); L. V. Keldysh, Sov. Phys.-JETP 20, 1018 (1965); V. Korenman, Ann. Phys. (N.Y.) 34, 72 (1966).

[50] L. P. Kadanoff and G. Baym, Quantum statistical mechanics (Benjamin, New York, 1962).

[51] R. P. Feynman, Phys. Rev. 80, 440 (1950).

[52] V. Ya. Fainberg, N. K. Pak, Teor. Mat. Fiz. 103, 328 (1995) [Theor. Math. Phys. 103, 595 (1995)].

[53] E. C. G. Stückelberg, Helv. Phys. Acta 15, 23 (1942).

[54] For a recent review see, e. g., D. J. Louis-Martinez, Phys. Lett. B 632, 733 (2006).

[55] I. S. Gradshteyn and I. M. Ryzhik, Tables of integrals, series, and products 6th ed. (Academic Press, San Diego, 2000). 